\title{
Oligo-Miocene extensional tectonics and fluid flow across the Northern Snake Range detachment system, Nevada
}

\section{Gebelin, A}

http://hdl.handle.net/10026.1/8622

\subsection{9/2010TC002797}

Tectonics

All content in PEARL is protected by copyright law. Author manuscripts are made available in accordance with publisher policies. Please cite only the published version using the details provided on the item record or document. In the absence of an open licence (e.g. Creative Commons), permissions for further reuse of content should be sought from the publisher or author. 


\title{
Oligo-Miocene extensional tectonics and fluid flow across the Northern Snake Range detachment system, Nevada
}

\author{
Aude Gébelin, ${ }^{1,2}$ Andreas Mulch, ${ }^{1,2,3}$ Christian Teyssier, ${ }^{4}$ Matt Heizler, ${ }^{5}$ \\ Torsten Vennemann, ${ }^{6}$ and Nicholas C. A. Seaton ${ }^{4}$ \\ Received 16 September 2010; revised 7 June 2011; accepted 14 July 2011; published 13 October 2011.
}

[1] The Northern Snake Range (Nevada) represents a spectacular example of a metamorphic core complex and exposes a complete section from the mylonitic footwall into the hanging wall of a fossil detachment system. Paired geochronological and stable isotopic data of mylonitic quartzite within the detachment footwall reveal that ductile deformation and infiltration of meteoric fluids occurred between 27 and $23 \mathrm{Ma} .{ }^{40} \mathrm{Ar} /{ }^{39} \mathrm{Ar}$ ages display complex recrystallization-cooling relationships but decrease systematically from $26.9 \pm 0.2 \mathrm{Ma}$ at the top to $21.3 \pm 0.2 \mathrm{Ma}$ at the bottom of footwall mylonite. Hydrogen isotope $(\delta \mathrm{D})$ values in white mica are very low $(-150$ to $-145 \%)$ within the top $80-90 \mathrm{~m}$ of detachment footwall, in contrast to values obtained from the deeper part of the section where values range from -77 to $-64 \%$, suggesting that time-integrated interaction between rock and meteoric fluid was restricted to the uppermost part of the mylonitic footwall. Pervasive mica-water hydrogen isotope exchange is difficult to reconcile with models of ${ }^{40} \mathrm{Ar}$ loss during mylonitization solely by volume diffusion. Rather, we interpret the ${ }^{40} \mathrm{Ar}{ }^{39} \mathrm{Ar}$ ages of white mica with low- $\delta \mathrm{D}$ values to date syn-mylonitic hydrogen and argon isotope exchange, and we conclude that the hydrothermal system of the Northern Snake Range was active during late Oligocene $(27-23 \mathrm{Ma})$ and has been exhumed by the combined effects of ductile strain, extensional detachment faulting, and erosion.

Citation: Gébelin, A., A. Mulch, C. Teyssier, M. Heizler, T. Vennemann, and N. C. A. Seaton (2011), Oligo-Miocene extensional tectonics and fluid flow across the Northern Snake Range detachment system, Nevada, Tectonics, 30, TC5010, doi:10.1029/2010TC002797.

\section{Introduction}

[2] Metamorphic core complexes (MCC) are bounded by brittle-to-ductile detachments that control lateral and vertical displacements and shape the topography of the Earth's surface during crustal-scale extension and mass transport in the middle and lower crust [e.g., Gans, 1987; Block and Royden, 1990; Teyssier et al., 2005; Mulch et al., 2006; Tirel et al., 2008]. MCCs or extensional gneiss domes are therefore common features of many of the world's largest orogens and represent important tectonic elements that record the geodynamic evolution of the Earth's crust during

\footnotetext{
${ }^{1}$ Institut für Geologie, Leibniz Universität Hannover, Hannover, Germany.

${ }^{2}$ Now at Biodiversity and Climate Research Centre (BiK-F), Frankfurt, Germany.

${ }^{3}$ Now at Goethe Universität Frankfurt, Frankfurt, Germany.

${ }^{4}$ Department of Geology and Geophysics, University of Minnesota, Minneapolis, Minnesota, USA.

${ }^{5}$ New Mexico Bureau of Geology and Mineral Resources, New Mexico Institute of Mining and Technology, Socorro, New Mexico, USA.

${ }^{6}$ Institute of Mineralogy and Geochemistry, University of Lausanne, Lausanne, Switzerland.

Copyright 2011 by the American Geophysical Union. 0278-7407/11/2010TC002797
}

orogeny. The mylonitic footwall of such detachments represents a zone of high strain that varies in thickness from 10 s to 100 s of meters and separates the cool upper crust from the hot middle crust. While active, this zone of localized deformation hosts very large temperature gradients [Rey et al., 2009a, 2009b] that may be transient [Mulch et al. 2006; Gottardi et al., 2011].

[3] The interplay between surface topography, heat advection via extensional fault systems, and orographic rainfall permits circulation of meteoric fluids in fault-controlled hydrothermal systems that can reach footwall depths corresponding to the brittle-ductile transition [e.g., Person et al., 2007]. The role of extensional detachment zones in the exhumation, retrograde metamorphism, and cooling of uplifted metamorphic rocks of the mylonitic footwall is relatively well understood [e.g., Rey et al., 2009b]. Yet, the impact of brittle normal faults and extensional ductile strain in the mylonitic footwall on the crustal-scale transport of fluids, the thermal and mechanical role of these fluids during mylonitization within and below the detachment, and the effect of detachment faults on the associated topography at the Earth's surface, is largely elusive [Fricke et al., 1992; Morrison and Anderson, 1998; Famin et al., 2004; Mulch and Chamberlain, 2007; Person et al., 2007]. 


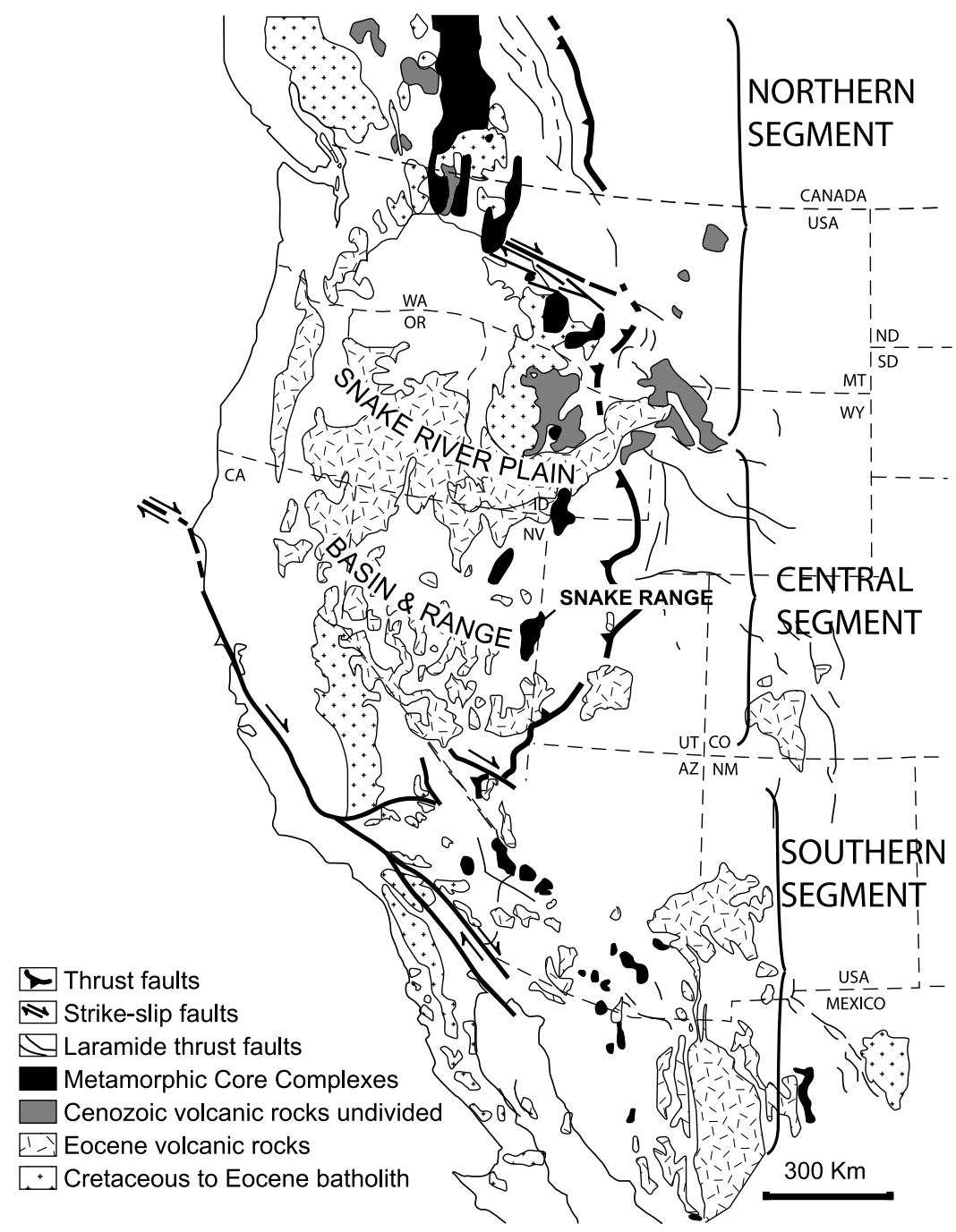

Figure 1. Distribution of Metamorphic Core Complexes in North American Cordillera.

[4] Here we present a study of an exhumed fossil detachment system that formed during Cenozoic extension of the North American Cordillera to characterize when surface fluids penetrated the crust, and how large-scale convective hydrothermal flow patterns affect detachment zone dynamics.

[5] The Northern Snake Range MCC (Nevada, USA) (Figure 1) is an excellent locality to assess the role of fluids in detachment systems because 1) it is well studied [e.g., Wernicke, 1981; Miller et al., 1983; Bartley and Wernicke, 1984; Lee and Sutter, 1991; Lee, 1995; Lewis et al., 1999; Miller et al., 1999a; Lee et al., 1987; Gans et al., 1985, 1989], 2) the upper crust experienced more than $450 \%$ extension [Miller et al., 1983] resulting in open brittle fractures that permit downward percolation of fluids [Losh, 1997], and 3) the region had a high geothermal gradient, which could drive fluid flow, because heat was advected within the footwall of the detachment, with some additional contribution from the emplacement of crustal melts ( $\sim 35 \mathrm{Ma}$, Eocene-Oligocene batholiths [Gans et al., 1989]).

[6] The eastern flank of the Northern Snake Range MCC exposes a complete section through a fossil detachment system that in its footwall is composed almost entirely of mylonitic quartzite and interlayered schist units [e.g., Lee et al., 1987; Lee, 1995; Miller et al., 1999a]. Oxygen and hydrogen isotope geochemistry represents an excellent tool to trace the fate of fluids in such brittle-ductile detachment systems [e.g., Wickham and Taylor, 1987; Fricke et al., 1992; Losh, 1997; Morrison and Anderson, 1998; Holk and Taylor, 2007; Mulch et al., 2004, 2007]. Equilibrium isotope exchange between water and hydrous minerals not only tracks the origin of fluids but also the timing and duration of isotopic exchange if growth of these minerals can be dated. Muscovite collected systematically across the Northern Snake Range detachment (NSRD) mylonitic footwall indicates a systematic pattern of $\delta \mathrm{D}$ values, ${ }^{40} \mathrm{Ar} /{ }^{39} \mathrm{Ar}$ ages, and quartz microstructures that we interpret to reflect the combined effects of exhumation-related footwall cooling, fluidrock interaction, and mineral growth and recrystallization. $\delta \mathrm{D}$ values of muscovite as low as $-150 \%$ (SMOW), reflecting a meteoric water source at the top of the detachment zone become progressively less negative over the $300 \mathrm{~m}$ thick section of exposed mylonite, reaching metamorphic $\delta \mathrm{D}$ values of $-64 \%$ at the deepest levels of footwall exposure. 


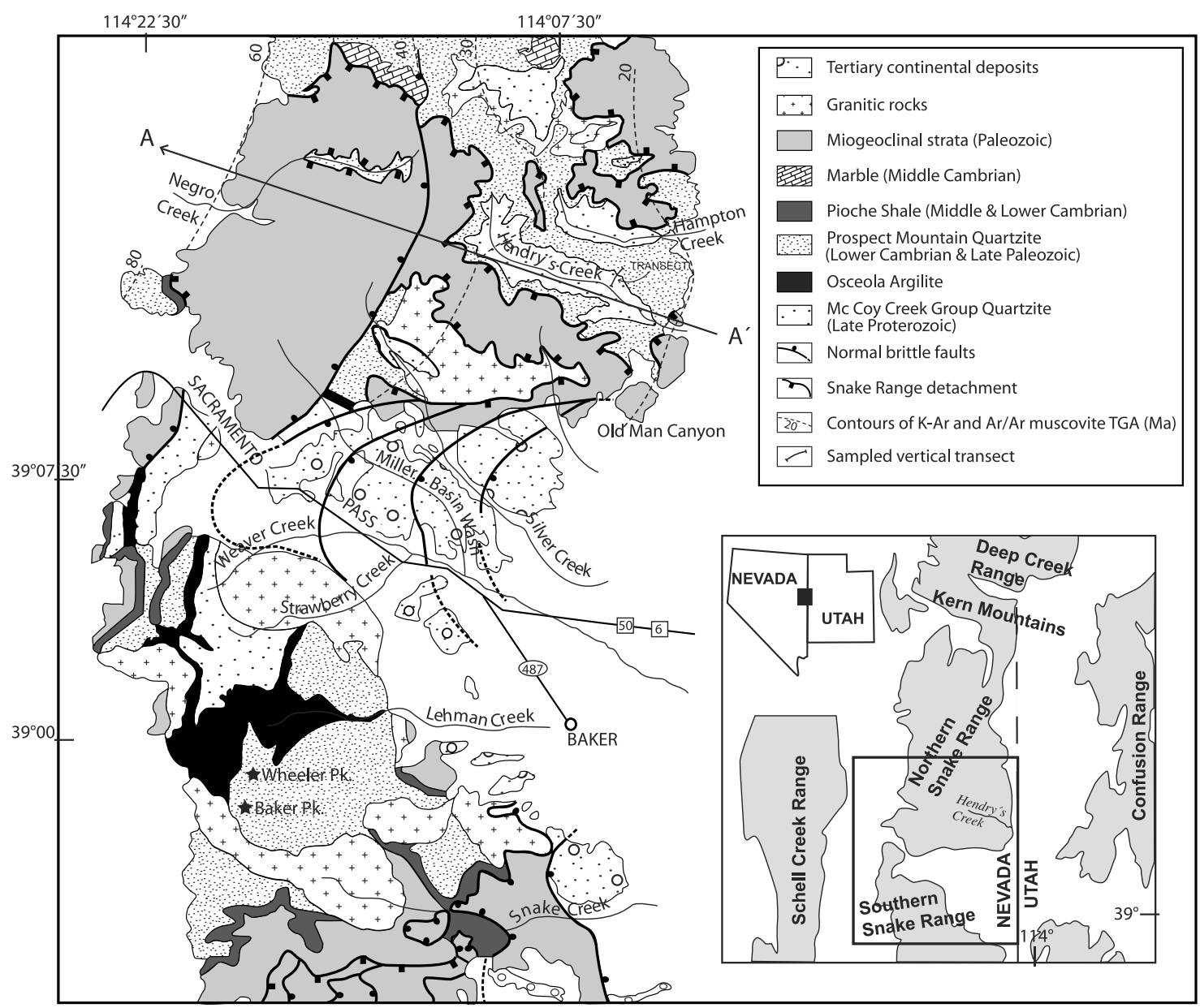

Figure 2. Geological map showing the Northern Snake Range Detachment along the eastern flank of the range and brittle normal faults in the upper plate. Also shown is the location of the vertical sampling transect and the total gas argon (TGA) K-Ar and Ar/Ar muscovite contours from Armstrong and Hansen [1966], Lee et al. [1970, 1980], and Lee and Sutter [1991].

Our reported ${ }^{40} \mathrm{Ar} /{ }^{39} \mathrm{Ar}$ muscovite ages (26.9 to $21.3 \mathrm{Ma}$ ) are in agreement with earlier data acquired by Lee and Sutter [1991]. However, we interpret the ${ }^{40} \mathrm{Ar} /{ }^{39} \mathrm{Ar}$ ages (26.9 to $23 \mathrm{Ma}$ ) of muscovite with low- $\delta \mathrm{D}$ values to reflect argon and hydrogen isotope exchange most likely by lattice-scale recrystallization of white mica rather than cooling through closure to argon diffusion.

\section{Geologic Setting}

[7] The Northern Snake Range MCC developed as a consequence of protracted Tertiary extension across the Basin and Range Province [e.g., Wernicke, 1981; Miller et al., 1983; Bartley and Wernicke, 1984; Gans et al., 1985, 1989; Lee and Sutter, 1991; Lewis et al., 1999; Miller et al., 1999a]. This core complex is bounded by the Northern Snake Range Detachment (NSRD) (Figure 2), which strikes N-S and extends over $150 \mathrm{~km}$ from the southern Snake Range to Kern Mountains and Deep Creek Range in the North [Gans et al., 1999a, 1999b; Lee et al., 1999a, 1999b, 1999c; Miller and Gans, 1999; Miller et al., 1999b).

[8] With a predominant top to the east sense of shear, deformation along the NSRD and its mylonitic footwall was responsible for exhumation of metamorphosed Upper Proterozoic to Lower Cambrian quartzite and metapelite and Middle Cambrian to Ordovician marble relative to normalfaulted Paleozoic and Tertiary strata in the hanging wall [Wernicke, 1981; Lee et al., 1987; Miller et al., 1999a] (Figures 2 and 3). The Snake Range MCC has been at the heart of a major debate about the general style of lithospheric extension, and in particular on the role of pure shear versus simple shear in crustal deformation. Wernicke [1981] interpreted the NSRD as a major Tertiary low-angle normal fault (décollement) that accommodated several tens and perhaps more than $60 \mathrm{~km}$ of extensional displacement [Bartley and Wernicke, 1984]. In contrast, Miller et al. [1983], Gans and Miller [1983], Gans et al. [1985], Lee et al. [1987], Miller and Gans [1989], and Lee and Sutter [1991] depict the NSRD as a subhorizontal brittle-ductile transition zone with only limited $(<10 \mathrm{~km})$ slip. The brittlely extended upper plate (450\%) rests on top of an equally stretched lower plate showing Cenozoic greenschist-facies metamorphism. In this "pure-shear" model, no significant structural repetition or omission of section exists across the NSRD. However, Miller et al. [1983] and Lee et al. [1987] also recognized a strong west-to-east increase in strain with 


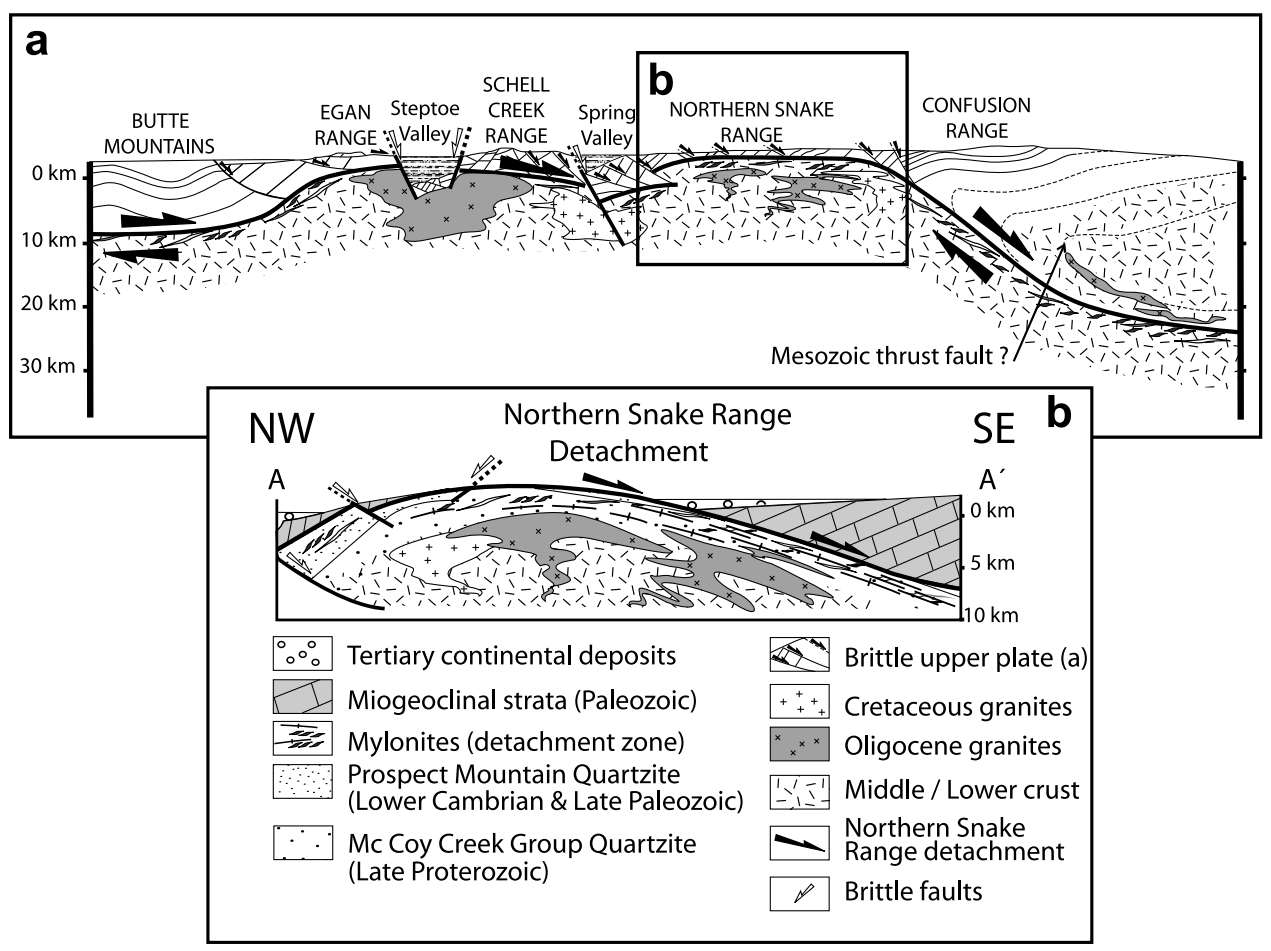

Figure 3. (a) Idealized cross section of east-central Nevada modified after Gans and Miller [1983] but including a rolling-hinge detachment that affects the Snake Range, Schell Creek Range and Egan Range. (b) Schematic NW-SE cross section across the Northern Snake Range modified after Miller et al. [1999a] showing the top-to-east NSRD that accommodates exhumation of Late Proterozoic, Cambrian and Ordovician metamorphic rocks from beneath upper Paleozoic and Tertiary strata. Section location in Figure 2.

a constant and dominant top-to-east non-coaxial strain component that can be identified in the eastern, and particularly in the southeastern part of the range. It is not the aim of this paper to re-evaluate competing models of crustal-scale extension; however, the data presented here are consistent with the model of a rolling-hinge-type extensional detachment that progressively exhumed parts of the mylonitic footwall (Figure 3).

[9] Moreover, ${ }^{40} \mathrm{Ar} /{ }^{39} \mathrm{Ar}$ thermochronology of muscovite, biotite, and potassium feldspar from mylonitic quartzite and schist in the NSRD footwall shows a systematic decrease of ages from $\sim 80 \mathrm{Ma}$ on the west flank to $\sim 20 \mathrm{Ma}$ on the east flank of the range [Lee and Sutter, 1991] (Figure 2). This age gradient suggests prolonged residence at higher temperatures of rocks in the east and asymmetric unroofing of the range. Based on diffusion-domain analysis of ${ }^{40} \mathrm{Ar} /{ }^{39} \mathrm{Ar}$ K-feldspar data, Lee [1995] calculated thermal histories and showed that the lower plate cooled below $300^{\circ} \mathrm{C}$ at $\sim 46 \mathrm{Ma}$ in the west and at $\sim 19 \mathrm{Ma}$ in the east.

[10] Together with apatite fission track data [Miller et al., 1993], Lee [1995] deduced a differential cooling history, including three episodes of rapid cooling $\left(10-55^{\circ} \mathrm{C} / \mathrm{Ma}\right)$ : (1) in the middle Eocene (48-41 Ma) related to the initiation of slip along the NSRD; (2) in the late Oligocene (30-26 Ma) attributed to a deformational pulse along the NSRD; and (3) during the early Miocene (20-16 Ma). The latter marks the cessation of ductile deformation within the detachment, a major pulse of uplift, and the rotation of lower plate rocks from beneath the NSRD. Based on this large body of ther- mochronological data, Lee [1995] interpreted the NSRD as a rolling-hinge detachment with a listric shape that rotated from steep $\left(>40^{\circ}\right)$ to shallow $\left(10^{\circ}-20^{\circ}\right)$ dips in response to denudation and isostatic unloading of the footwall. Building upon the work of Lee [1995], who proposed an age of $20 \mathrm{Ma}$ for the cessation of the NSRD activity, zircon and apatite fission track data obtained by Miller et al. [1999a] suggest that parts of the detachment were still active until $17 \mathrm{Ma}$ along the eastern flank of the range where at least $12-15 \mathrm{~km}$ of displacement have been accommodated. Miller et al. [1999a] suggested lower crustal flow and upwelling of rocks during initial extension (35-20 Ma) was followed by significant slip on the detachment and ultimate unroofing of the lower plate in the middle Miocene ( $\sim 17 \mathrm{Ma})$.

[11] The exact mode of extension necessarily plays a role for detachment-controlled fluid circulation. Despite a lack of consensus, there is general agreement that the footwall to the NSRD cuts across an exhumed brittle-ductile transition zone and that the footwall of the NSRD along the eastern flank of the Snake Range in the region of Hendry's Creek represents a top-to-east mylonitic shear zone (Figures 2 and 3).

\section{Hendry's Creek}

[12] We systematically sampled mylonitic quartzite and schist across ca. $300 \mathrm{~m}$ of structural section from the NSRD into the underlying mylonitic footwall at Hendry's Creek (Figure 2), which deeply incises Upper Proterozoic (McCoy Creek) and Lower Cambrian (Prospect Mountain) 


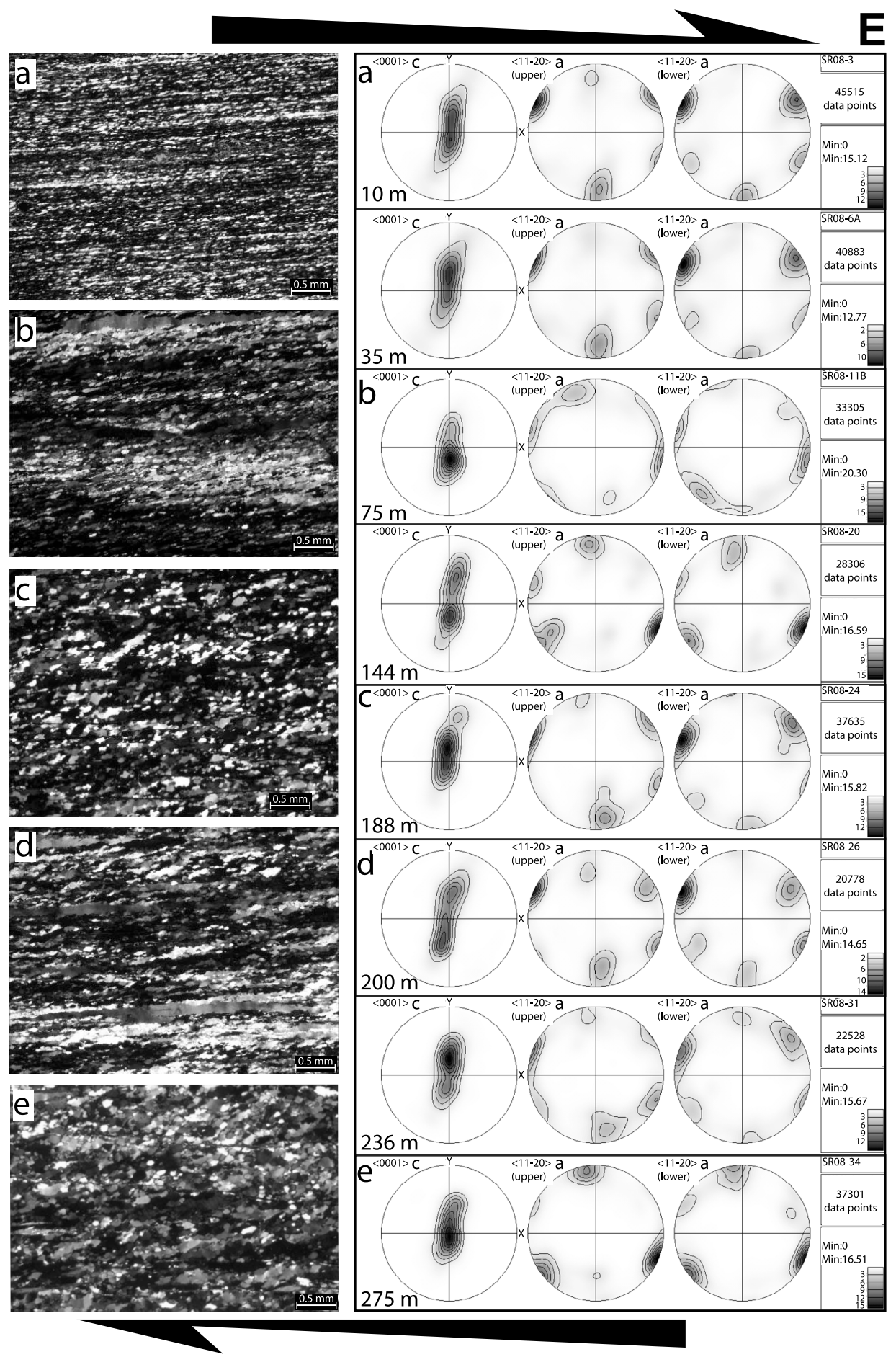

Figure 4. (a-e) Quartz microstructures and LPO measured using EBSD. Equal-area projection, upper hemisphere. Foliation (XY plane) is vertical, and lineation (X) is horizontal in this plane. See text for description.

quartzite and schist units (Figures 2, 3 and structural section in Figure 7). The mylonitic rocks contain a shallowly E-dipping foliation and a WNW - ESE trending stretching lineation. The light gray to white, fine-grained Prospect Mountain quartzite forms the top $60 \mathrm{~m}$ of the section. This unit conformably overlies the metamorphosed Osceola
Argillite [Miller et al., 1995]. The top of the McCoy Creek Group beneath the Osceola Argillite is characterized by fineto medium-grained quartzite about $25-30 \mathrm{~m}$ thick that overlies a garnet-bearing schist (see stratigraphic column on Figure 7). This schist is interlayered with $\sim 70 \mathrm{~m}$ of mediumto coarse-grained quartzite. A $\sim 25 \mathrm{~m}$ thick staurolite-garnet 

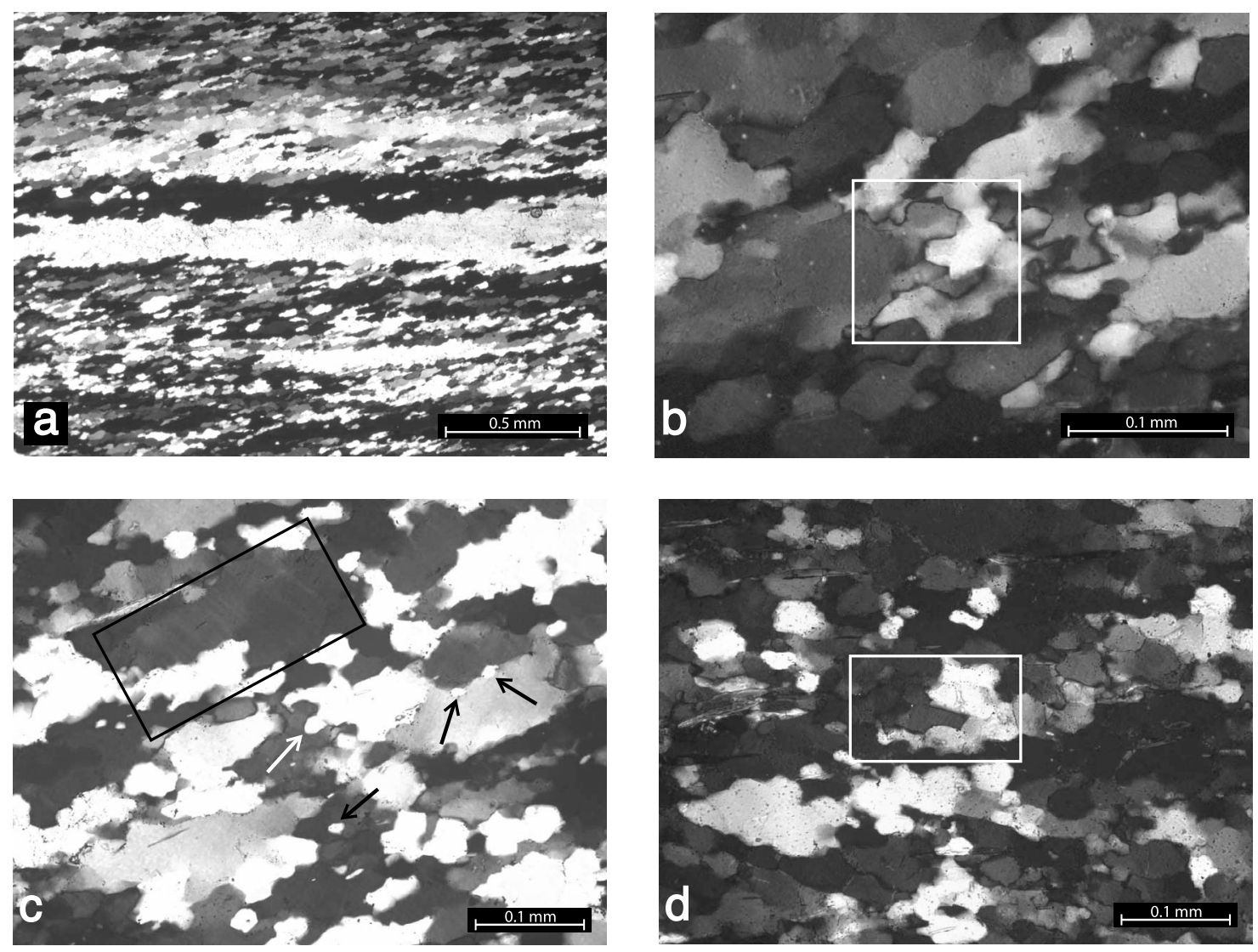

Figure 5. (a) Microstructures illustrating recrystallization by progressive subgrain rotation. Large original quartz grains are flattened and exhibit recrystallized grains along their grain boundaries or display complete recrystallization. (b) Larger quartz grain sharing castellate boundaries with two or three smaller quartz grains (sample SR08-24). (c) Fingers of one grain consuming areas of a neighboring grain resulting in the formation of isolated grains (arrows). Deformation lamellae in large quartz grain (black square), (sample SR08-34). (d) Pinning microstructure (SR08-35).

schist rests on top of a massive quartzite unit, which forms the deepest unit exposed along Hendry's Creek.

\section{Quartzite Microstructure and Electron Backscatter Diffraction (EBSD) Analysis}

[13] We examined 35 thin sections distributed evenly (spacing $\sim 10 \mathrm{~m}$ ) over the Hendry's Creek section and chose eight samples of mylonitic quartzite for quartz microfabric analysis using EBSD (Figure 4) (analytical procedure in Appendix A). These studied thin sections are distributed over the exposed $300 \mathrm{~m}$ of the NSRD footwall, with the contact with the NSRD as reference level. The top $60 \mathrm{~m}$ of mylonitic Prospect Mountain quartzite are characterized by a very small $(5$ to $25 \mu \mathrm{m})$ recrystallized quartz grain size (Figure $4 \mathrm{a}$ ). These quartz grains are elongate at an angle of about $25^{\circ}$ from the macroscopic (field measured) foliation which in thin section is defined by the surfaces that separate mica fish (shear plane). The fabric asymmetry indicates topto-east sense of shear. Quartz c-axis fabrics display a preferred orientation parallel to the $y$ axis of the finite strain ellipsoid (X-Y-Z are the long, intermediate, and short principal axes of finite strain ellipsoid) and a single-girdle shape that is asymmetric with respect to the finite strain axes
(Figure 4a), indicating top-to-east sense of shear; quartz aaxis are distributed asymmetrically around the stretching lineation, with a maximum indicating east-directed shear. Such microfabrics are compatible with deformation in the dislocation creep regime dominated by prism $\langle\mathrm{a}\rangle$ glide [Bouchez, 1977; Law, 1990].

[14] Between 60 and $145 \mathrm{~m}$ of section, quartz grains display intense dynamic recrystallization (Figure $4 \mathrm{~b}$ ), are elongate obliquely to the shear plane and on average have a larger recrystallized grain size $(\sim 40 \mu \mathrm{m})$ compared to the top of the section. These grains are likely to have recrystallized by subgrain rotation from large elongate quartz grains $(>1 \mathrm{~mm})$ oriented parallel to the shear plane (Figures $4 \mathrm{~b}$ and $5 \mathrm{a}$ ). A strong point maximum of $\mathrm{c}$-axes parallel to the $y$ axis of the finite strain ellipsoid is consistent with prism $\langle a\rangle$ slip and the concentration of a-axes at $10-15^{\circ}$ to the shear plane again indicates top-to-east sense of shear (Figure 4b).

[15] Between 145 and $188 \mathrm{~m}$ the mylonitic quartz matrix is homogeneously recrystallized (Figure 4c) with an average grain size of $\sim 50 \mu \mathrm{m}$. Castellate microstructure (Figure 5b), pinning of quartz grain boundaries by mica grains, and inclusion of small, foliation-parallel mica grains within quartz grains suggest that grain boundary migration was the dominant dynamic recrystallization process [Jessell, 1987]. 


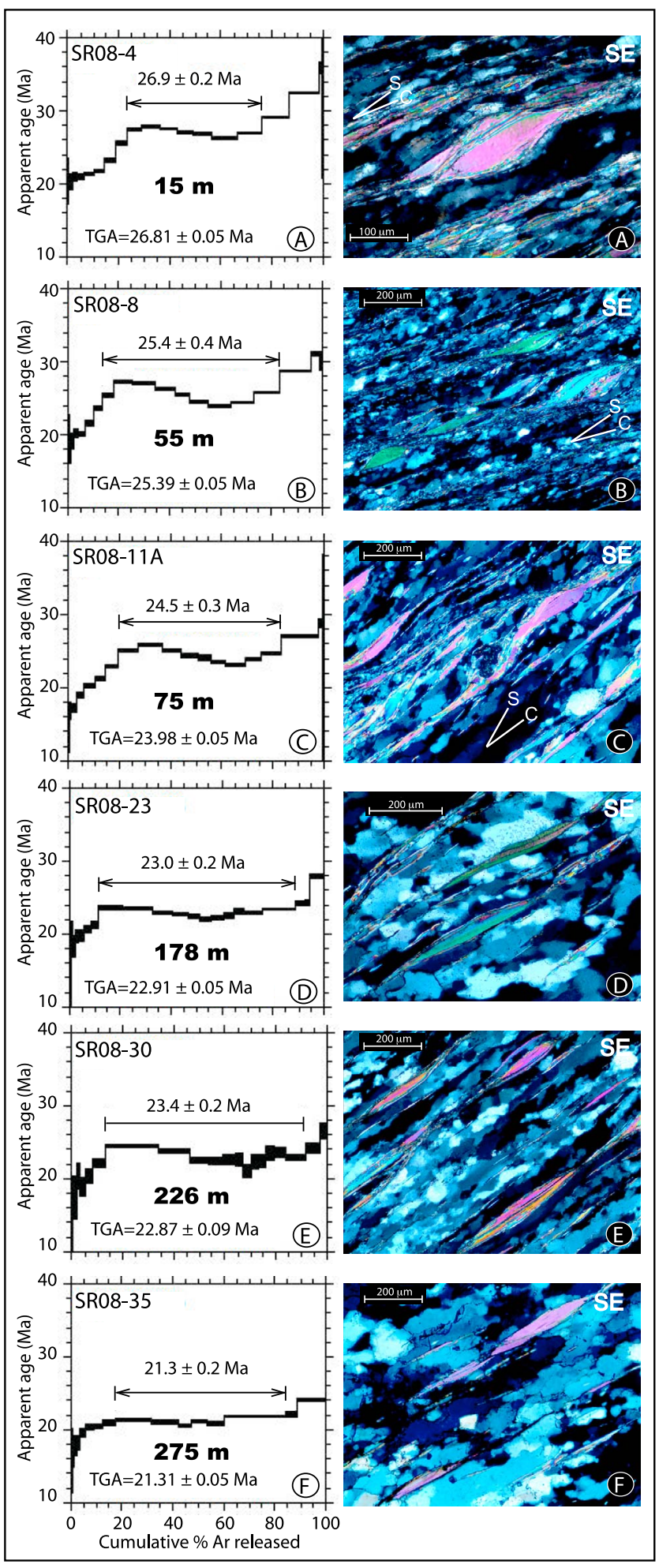

Figure 6. (a-f) $\mathrm{The}^{40} \mathrm{Ar} /{ }^{39} \mathrm{Ar}$ step-heating spectra of muscovite fish (muscovite microstructures on the right) from six mylonitic quartzites distributed over the Snake Range detachment footwall.

Recrystallized grains are elongate and oblique to the shear plane. Quartz c-axes form an asymmetric girdle and display a marked concentration near the $y$ axis of the finite strain ellipsoid (Figure 4c). Quartz a-axes have a maximum at $25^{\circ}$ to the shear plane again in agreement with the top-to-east sense of shear.

[16] Below $188 \mathrm{~m}$, relics of large quartz grains are abundant (Figure 4d) and mylonitic quartzite is characterized by a large range of recrystallized grain sizes ( $40 \mu \mathrm{m}$ to more than $200 \mu \mathrm{m}$; Figures $4 \mathrm{~d}$ and $4 \mathrm{e}$ ). Large quartz grains commonly show deformation lamellae indicative of high shear stress and the dearth of effective recovery mechanisms [Hirth and Tullis, 1992] (Figure 5c). Window microstructures or isolated grains (i.e., "left-over grains" of Jessell [1987]; Figure 5c) indicate that smaller quartz formed by grain boundary migration. Castellate or pinning microstructures (Figure 5d) support this interpretation. The quartz c-axis fabric is defined by a strong $y$ axis parallel maximum that stretches into an inclined girdle, whose pole is the largest concentration of a-axes, consistent with top-to-east shearing.

[17] In summary, quartz c-axis and a-axes fabrics are practically constant from the top to the base of the section and indicate a strong component of non-coaxial deformation (Figure 4). A-axes are distributed asymmetrically with respect to the lineation direction, and c-axes exhibit a strong $y$ axis parallel maximum and an oblique partial single girdle indicating top-to-east sense of shear. Based solely on quartz microstructure observations, these data are compatible with plastic deformation under relatively high stress (and likely strain rate), dominated by prism $\langle$ a $\rangle$ glide that typically occurs between $400^{\circ} \mathrm{C}$ and $700^{\circ} \mathrm{C}$ [Tullis et al., 1973; Mainprice and Paterson, 1984]. Recrystallization by subgrain rotation (regime 2 [Hirth and Tullis, 1992]) and grain boundary migration (regime 3 ) are the principal mechanisms that can occur over a wide temperature range $\left(400\right.$ to $\left.\geq 550^{\circ} \mathrm{C}\right)$ at higher or lower stress, respectively. The transition from regime 2 to regime 3 occurs with increasing temperature and decreasing strain rate [Hirth and Tullis, 1992]. Based on the observed constant orientation of foliation and lineation, c-axis fabrics and kinematics, we propose that these regimes were active during the development of the detachment footwall mylonite and that they correspond to a single (protracted) strain and kinematic event.

\section{Muscovite Microstructure and ${ }^{40} \mathrm{Ar} /{ }^{39} \mathrm{Ar}$ Geochronology}

\subsection{Muscovite Microstructure}

[18] Muscovite collected from the top $83 \mathrm{~m}$ of the section at Hendry's Creek (Figures 6a, 6b, and 6c) is abundant $(\sim 30 \%)$ and displays elongate $(\geq 150 \times 40 \mu \mathrm{m})$, lozengeshape grains (muscovite fish). Long axes of the muscovite grains are inclined and parallel the elongate quartz grain shape fabric that makes an angle of $\sim 25^{\circ}$ to the shear plane. Mica fish commonly display very small muscovite grains along the rims and in pressure shadows. Trails of fine mica extending from the tips of oblique, isolated mica-fish form stair-stepping patterns that are consistent with top-to-east sense of shear [Lister and Snoke, 1984].

[19] Following the mica fish morphological grouping proposed by ten Grotenhuis et al. [2003], mica grains in the NSRD footwall form lenticular mica fish (group 1) and, toward the very top of the mylonitic footwall, cleavage planes that have sigmoidal shapes converging at one or both tips of the mica grains (group 2). Migration recrystallization 
Table 1a. Furnace Step Heating ${ }^{40} \mathrm{Ar} /{ }^{39} \mathrm{Ar}$ Data of Muscovite From the Northern Snake Range Detachment Footwall, Nevada

ID Temperature $\left({ }^{\circ} \mathrm{C}\right){ }^{40} \mathrm{Ar} /{ }^{39} \mathrm{Ar} \quad{ }^{37} \mathrm{Ar} /{ }^{39} \mathrm{Ar}{ }^{36} \mathrm{Ar} /{ }^{39} \mathrm{Ar}\left(\mathrm{x} 10^{-3}\right){ }^{39} \mathrm{Ar} \mathrm{K}_{\mathrm{K}}\left(\mathrm{x} 10^{-15} \mathrm{~mol}\right) \quad \mathrm{K} / \mathrm{Ca} \quad{ }^{40} \mathrm{Ar} *(\%) \quad{ }^{39} \mathrm{Ar}(\%) \quad$ Age $(\mathrm{Ma}) \quad \pm 1 \sigma(\mathrm{Ma})$

\begin{tabular}{|c|c|c|c|c|c|c|c|c|c|c|}
\hline \multicolumn{11}{|c|}{ SR08-4, White Mica, $5.46 \mathrm{mg}, J=0.0022937 \pm 0.07 \%, D=1.004 \pm 0.001, N M-223 B, L a b \#=58883-01$} \\
\hline A & 500 & 17.09 & 0.029 & 42.135 & 1.793 & 17.75 & 27.1 & 0.6 & 19.07 & 1.03 \\
\hline B & 550 & 10.18 & 0.015 & 16.780 & 1.285 & 34.27 & 51.3 & 1.1 & 21.46 & 1.03 \\
\hline $\mathrm{C}$ & 600 & 7.60 & 0.009 & 9.002 & 2.347 & 59.32 & 65.0 & 1.9 & 20.29 & 0.54 \\
\hline $\mathrm{D}$ & 650 & 6.55 & 0.001 & 4.921 & 4.517 & 805.25 & 77.8 & 3.5 & 20.94 & 0.29 \\
\hline $\mathrm{E}$ & 700 & 5.98 & 0.001 & 3.006 & 8.139 & 348.50 & 85.1 & 6.3 & 20.91 & 0.16 \\
\hline $\mathrm{F}$ & 750 & 5.83 & 0.001 & 2.207 & 12.573 & 656.97 & 88.8 & 10.8 & 21.26 & 0.13 \\
\hline $\mathrm{G}$ & 780 & 5.98 & 0.001 & 2.350 & 11.628 & 434.66 & 88.4 & 14.8 & 21.69 & 0.13 \\
\hline $\mathrm{H}$ & 800 & 6.31 & 0.001 & 2.236 & 11.551 & 758.44 & 89.5 & 18.9 & 23.17 & 0.12 \\
\hline I & 820 & 6.84 & 0.000 & 2.227 & 13.049 & 2616.43 & 90.4 & 23.5 & 25.38 & 0.11 \\
\hline $\mathrm{J}$ & 840 & 7.43 & 0.000 & 2.589 & 16.492 & 1144.73 & 89.7 & 29.3 & 27.34 & 0.12 \\
\hline K & 860 & 7.41 & 0.001 & 2.206 & 19.406 & 775.03 & 91.2 & 36.1 & 27.72 & 0.09 \\
\hline $\mathrm{L}$ & 880 & 7.16 & 0.000 & 1.636 & 20.008 & 2274.65 & 93.2 & 43.1 & 27.37 & 0.08 \\
\hline M & 900 & 7.07 & 0.001 & 1.744 & 18.112 & 834.89 & 92.7 & 49.4 & 26.90 & 0.10 \\
\hline $\mathrm{N}$ & 930 & 7.08 & 0.000 & 1.957 & 21.270 & 1101.24 & 91.8 & 56.9 & 26.66 & 0.09 \\
\hline $\mathrm{O}$ & 960 & 6.99 & 0.000 & 2.150 & 27.623 & 1222.92 & 90.9 & 66.6 & 26.05 & 0.08 \\
\hline $\mathrm{P}$ & 990 & 7.20 & 0.000 & 2.211 & 28.338 & 1199.92 & 90.9 & 76.6 & 26.83 & 0.07 \\
\hline Q & 1020 & 7.65 & 0.000 & 1.972 & 30.486 & 9572.31 & 92.4 & 87.3 & 28.99 & 0.08 \\
\hline $\mathrm{R}$ & 1060 & 8.27 & 0.000 & 1.167 & 32.199 & 1060.94 & 95.8 & 98.6 & 32.44 & 0.08 \\
\hline $\mathrm{S}$ & 1090 & 9.99 & 0.006 & 4.217 & 3.958 & 86.77 & 87.6 & 99.9 & 35.84 & 0.35 \\
\hline $\mathrm{T}$ & 1120 & 11.77 & 0.065 & 13.575 & 0.170 & 7.82 & 66.0 & 100.0 & 31.84 & 5.61 \\
\hline Int & age $\pm 1 \sigma$ & & $\mathrm{n}=20$ & & 284.946 & & & $\mathrm{~K} 2 \mathrm{O}=8.74 \%$ & 26.81 & 0.05 \\
\hline Pla & $1 \sigma$ & steps J-P & $\mathrm{n}=7$ & MSWD $=39.69$ & 151.249 & & & 53.1 & 26.95 & 0.21 \\
\hline
\end{tabular}

$\begin{array}{lc}\mathrm{A} & 500 \\ \mathrm{~B} & 550 \\ \mathrm{C} & 600 \\ \mathrm{D} & 650 \\ \mathrm{E} & 700 \\ \mathrm{~F} & 750 \\ \mathrm{G} & 780 \\ \mathrm{H} & 800 \\ \mathrm{I} & 820 \\ \mathrm{~J} & 840 \\ \mathrm{~K} & 860 \\ \mathrm{~L} & 880 \\ \mathrm{M} & 900 \\ \mathrm{~N} & 930 \\ \mathrm{O} & 960 \\ \mathrm{P} & 990 \\ \mathrm{Q} & 1020 \\ \mathrm{R} & 1060 \\ \mathrm{~S} & 1090 \\ \mathrm{~T} & 1120 \\ \text { Integrated age } \pm 1\end{array}$

Plateau $\pm 1 \sigma$

SR08-8, White Mica, $3.23 \mathrm{mg}, \mathrm{J}=0.0022972 \pm 0.10 \%, \mathrm{D}=1.004 \pm 0.001, \mathrm{NM}-223 \mathrm{~B}, \mathrm{Lab \#}=58882-01$

$\begin{array}{lllllllll}16.15 & 0.077 & 42.308 & 0.880 & 6.64 & 22.7 & 0.4 & 15.10 & 1.64 \\ 6.26 & 0.057 & 5.212 & 0.655 & 8.93 & 75.5 & 0.7 & 19.45 & 1.67 \\ 5.68 & 0.052 & 4.732 & 1.247 & 9.80 & 75.4 & 1.3 & 17.62 & 0.89 \\ 5.42 & 0.003 & 2.700 & 2.390 & 152.10 & 85.3 & 2.5 & 19.00 & 0.54 \\ 5.32 & 0.003 & 1.507 & 4.057 & 148.80 & 91.6 & 4.5 & 20.05 & 0.30 \\ 5.37 & 0.002 & 1.709 & 6.075 & 251.38 & 90.6 & 7.4 & 20.00 & 0.19 \\ 5.68 & 0.001 & 1.559 & 6.415 & 474.70 & 91.9 & 10.5 & 21.45 & 0.18 \\ 6.09 & 0.000 & 1.164 & 7.234 & 1284.50 & 94.3 & 14.1 & 23.60 & 0.18 \\ 6.50 & 0.000 & 1.255 & 9.800 & 1780.82 & 94.3 & 18.8 & 25.19 & 0.14 \\ 6.89 & 0.000 & 1.048 & 14.905 & - & 95.5 & 26.0 & 27.03 & 0.10 \\ 6.74 & 0.000 & 0.594 & 18.031 & 1219.71 & 97.4 & 34.8 & 26.96 & 0.08 \\ 6.47 & 0.001 & 0.426 & 15.604 & 664.07 & 98.1 & 42.4 & 26.08 & 0.09 \\ 6.29 & 0.000 & 0.514 & 12.699 & - & 97.6 & 48.5 & 25.22 & 0.10 \\ 6.17 & 0.000 & 0.743 & 14.848 & 1501.48 & 96.4 & 55.7 & 24.45 & 0.10 \\ 6.07 & 0.000 & 0.893 & 18.010 & - & 95.6 & 64.5 & 23.86 & 0.08 \\ 6.23 & 0.000 & 0.998 & 18.455 & - & 95.3 & 73.4 & 24.38 & 0.08 \\ 6.46 & 0.000 & 0.763 & 21.058 & 1859.34 & 96.5 & 83.7 & 25.62 & 0.08 \\ 7.10 & 0.000 & 0.445 & 25.178 & - & 98.1 & 95.9 & 28.61 & 0.07 \\ 8.19 & 0.001 & 2.210 & 6.840 & 725.96 & 92.1 & 99.2 & 30.96 & 0.17 \\ 7.34 & 0.001 & 0.145 & 1.639 & 377.31 & 99.4 & 100.0 & 29.94 & 0.66 \\ & \mathrm{n}=20 & & 206.021 & & & \mathrm{~K} 2 \mathrm{O}=10.67 \% & 25.39 & 0.05 \\ \text { steps I-Q } & \mathrm{n}=9 & \text { MSWD }=162.25 & 143.411 & & & 69.6 & 25.40 & 0.39\end{array}$

\begin{tabular}{|c|c|c|c|c|c|c|c|c|c|c|}
\hline A & 500 & 17.66 & 0.063 & 54.664 & 1.002 & 8.06 & 8.6 & 0.4 & 6.22 & 1.73 \\
\hline B & 550 & 6.71 & 0.001 & 10.937 & 0.842 & 609.85 & 51.7 & 0.8 & 14.19 & 1.50 \\
\hline C & 600 & 5.54 & -0.002 & 4.908 & 1.904 & - & 73.8 & 1.7 & 16.70 & 0.60 \\
\hline D & 650 & 5.41 & -0.005 & 4.132 & 3.774 & - & 77.4 & 3.4 & 17.11 & 0.34 \\
\hline E & 700 & 5.28 & -0.001 & 2.244 & 6.529 & - & 87.4 & 6.3 & 18.85 & 0.20 \\
\hline F & 750 & 5.47 & 0.000 & 1.781 & 9.876 & - & 90.4 & 10.7 & 20.19 & 0.14 \\
\hline G & 780 & 5.78 & 0.000 & 2.010 & 9.043 & - & 89.7 & 14.7 & 21.14 & 0.15 \\
\hline $\mathrm{H}$ & 800 & 6.36 & -0.001 & 2.530 & 11.566 & - & 88.2 & 19.9 & 22.91 & 0.13 \\
\hline I & 820 & 6.87 & 0.000 & 2.539 & 17.035 & - & 89.1 & 27.5 & 24.95 & 0.10 \\
\hline J & 840 & 6.77 & 0.000 & 1.607 & 20.354 & - & 93.0 & 36.6 & 25.67 & 0.08 \\
\hline K & 860 & 6.43 & 0.000 & 1.090 & 17.635 & - & 95.0 & 44.5 & 24.92 & 0.09 \\
\hline L & 880 & 6.30 & 0.000 & 1.151 & 14.718 & - & 94.6 & 51.1 & 24.30 & 0.10 \\
\hline M & 900 & 6.26 & 0.000 & 1.277 & 12.045 & - & 94.0 & 56.5 & 23.99 & 0.12 \\
\hline $\mathrm{N}$ & 930 & 6.26 & -0.001 & 1.721 & 12.890 & - & 91.9 & 62.2 & 23.47 & 0.12 \\
\hline $\mathrm{O}$ & 960 & 6.20 & 0.001 & 1.794 & 15.585 & 652.18 & 91.4 & 69.2 & 23.12 & 0.11 \\
\hline P & 990 & 6.41 & 0.002 & 1.877 & 13.966 & 282.74 & 91.3 & 75.5 & 23.89 & 0.11 \\
\hline Q & 1020 & 6.58 & 0.002 & 1.907 & 18.866 & 260.59 & 91.4 & 83.9 & 24.52 & 0.09 \\
\hline $\mathrm{R}$ & 1060 & 6.88 & 0.000 & 0.950 & 31.046 & - & 95.9 & 97.8 & 26.90 & 0.07 \\
\hline $\mathrm{S}$ & 1090 & 7.86 & 0.000 & 2.766 & 4.870 & 1328.31 & 89.7 & 100.0 & 28.72 & 0.26 \\
\hline $\mathrm{T}$ & 1120 & 6.57 & 0.068 & 7.308 & 0.111 & 7.45 & 67.2 & 100.0 & 18.05 & 10.07 \\
\hline \multirow{2}{*}{\multicolumn{2}{|c|}{ Integrated age $\pm 1 \sigma$}} & & $\mathrm{n}=20$ & & 223.657 & & & $\mathrm{~K} 2 \mathrm{O}=8.00 \%$ & 23.98 & 0.05 \\
\hline & & steps I-Q & $\mathrm{n}=9$ & MSWD $=66.58$ & 143.094 & & & 64.0 & 24.49 & 0.27 \\
\hline
\end{tabular}

SR08-11A, White Mica, $4.71 \mathrm{mg}, J=0.002279 \pm 0.09 \%, D=1.004 \pm 0.001, N M-223 \mathrm{~A}$, Lab\# $=58881-01$ 
Table 1a. (continued)

\begin{tabular}{|c|c|c|c|c|c|c|c|c|c|c|}
\hline ID & Temperature $\left({ }^{\circ} \mathrm{C}\right)$ & ${ }^{40} \mathrm{Ar} /{ }^{39} \mathrm{Ar}$ & ${ }^{37} \mathrm{Ar} /{ }^{39} \mathrm{Ar}$ & ${ }^{36} \mathrm{Ar} /{ }^{39} \operatorname{Ar}\left(\mathrm{x} 10^{-3}\right)$ & ${ }^{39} \mathrm{Ar}_{\mathrm{K}}\left(\mathrm{x} 10^{-15} \mathrm{~mol}\right)$ & $\mathrm{K} / \mathrm{Ca}$ & ${ }^{40} \mathrm{Ar}^{*}(\%)$ & ${ }^{39} \operatorname{Ar}(\%)$ & Age (Ma) & $\pm 1 \sigma(\mathrm{Ma})$ \\
\hline \multicolumn{11}{|c|}{ SR08-23, White Mica, $4.89 \mathrm{mg}, J=0.0022823 \pm 0.08 \%, D=1.004 \pm 0.001, N M-223 \mathrm{~A}$, Lab\# $=58880-01$} \\
\hline A & 500 & 13.11 & 0.090 & 35.381 & 2.018 & 5.69 & 20.3 & 1.0 & 10.90 & 0.86 \\
\hline B & 550 & 8.10 & 0.178 & 11.124 & 1.303 & 2.87 & 59.6 & 1.6 & 19.75 & 0.93 \\
\hline $\mathrm{C}$ & 600 & 6.95 & 0.089 & 8.452 & 1.860 & 5.76 & 64.1 & 2.5 & 18.22 & 0.79 \\
\hline $\mathrm{D}$ & 650 & 6.43 & 0.022 & 5.532 & 2.833 & 23.17 & 74.6 & 3.8 & 19.61 & 0.49 \\
\hline $\mathrm{E}$ & 700 & 6.32 & 0.009 & 5.034 & 3.932 & 56.20 & 76.4 & 5.7 & 19.74 & 0.38 \\
\hline $\mathrm{F}$ & 750 & 6.56 & 0.007 & 5.131 & 5.933 & 72.18 & 76.9 & 8.6 & 20.60 & 0.25 \\
\hline $\mathrm{G}$ & 780 & 6.73 & 0.007 & 5.241 & 7.046 & 70.55 & 77.0 & 11.9 & 21.18 & 0.22 \\
\hline $\mathrm{H}$ & 820 & 6.94 & 0.003 & 3.912 & 19.632 & 167.95 & 83.3 & 21.4 & 23.60 & 0.11 \\
\hline I & 840 & 6.39 & 0.002 & 2.098 & 23.724 & 275.52 & 90.3 & 32.7 & 23.56 & 0.08 \\
\hline $\mathrm{J}$ & 860 & 6.03 & 0.002 & 1.434 & 17.156 & 269.39 & 93.0 & 40.9 & 22.89 & 0.09 \\
\hline K & 880 & 5.98 & 0.003 & 1.412 & 12.588 & 195.38 & 93.0 & 47.0 & 22.70 & 0.11 \\
\hline $\mathrm{L}$ & 900 & 5.99 & 0.002 & 1.744 & 10.104 & 292.78 & 91.4 & 51.8 & 22.38 & 0.15 \\
\hline $\mathrm{M}$ & 930 & 6.03 & 0.002 & 2.148 & 9.713 & 235.54 & 89.5 & 56.5 & 22.04 & 0.15 \\
\hline $\mathrm{N}$ & 960 & 6.09 & 0.003 & 2.301 & 8.785 & 148.89 & 88.8 & 60.7 & 22.11 & 0.15 \\
\hline $\mathrm{O}$ & 990 & 6.19 & 0.002 & 2.303 & 8.383 & 251.23 & 89.0 & 64.7 & 22.50 & 0.16 \\
\hline $\mathrm{P}$ & 1020 & 6.25 & 0.003 & 2.008 & 8.554 & 170.59 & 90.5 & 68.8 & 23.11 & 0.16 \\
\hline Q & 1060 & 6.27 & 0.002 & 2.278 & 16.133 & 339.43 & 89.2 & 76.5 & 22.85 & 0.10 \\
\hline $\mathrm{R}$ & 1090 & 6.20 & 0.001 & 1.630 & 25.895 & 381.46 & 92.2 & 89.0 & 23.37 & 0.08 \\
\hline $\mathrm{S}$ & 1120 & 6.19 & 0.002 & 0.882 & 12.003 & 271.13 & 95.8 & 94.7 & 24.22 & 0.12 \\
\hline $\mathrm{T}$ & 1570 & 7.75 & 0.047 & 3.135 & 11.049 & 10.82 & 88.1 & 100.0 & 27.87 & 0.15 \\
\hline \multicolumn{3}{|c|}{ Integrated age $\pm 1 \sigma$} & $\mathrm{n}=20$ & & 208.644 & & & $\mathrm{~K} 2 \mathrm{O}=7.18 \%$ & 22.91 & 0.05 \\
\hline Plat & $\mathrm{eau} \pm 1 \sigma$ & steps H-R & $\mathrm{n}=11$ & $\mathrm{MSWD}=21.40$ & 160.667 & & & 77.0 & 23.01 & 0.15 \\
\hline
\end{tabular}

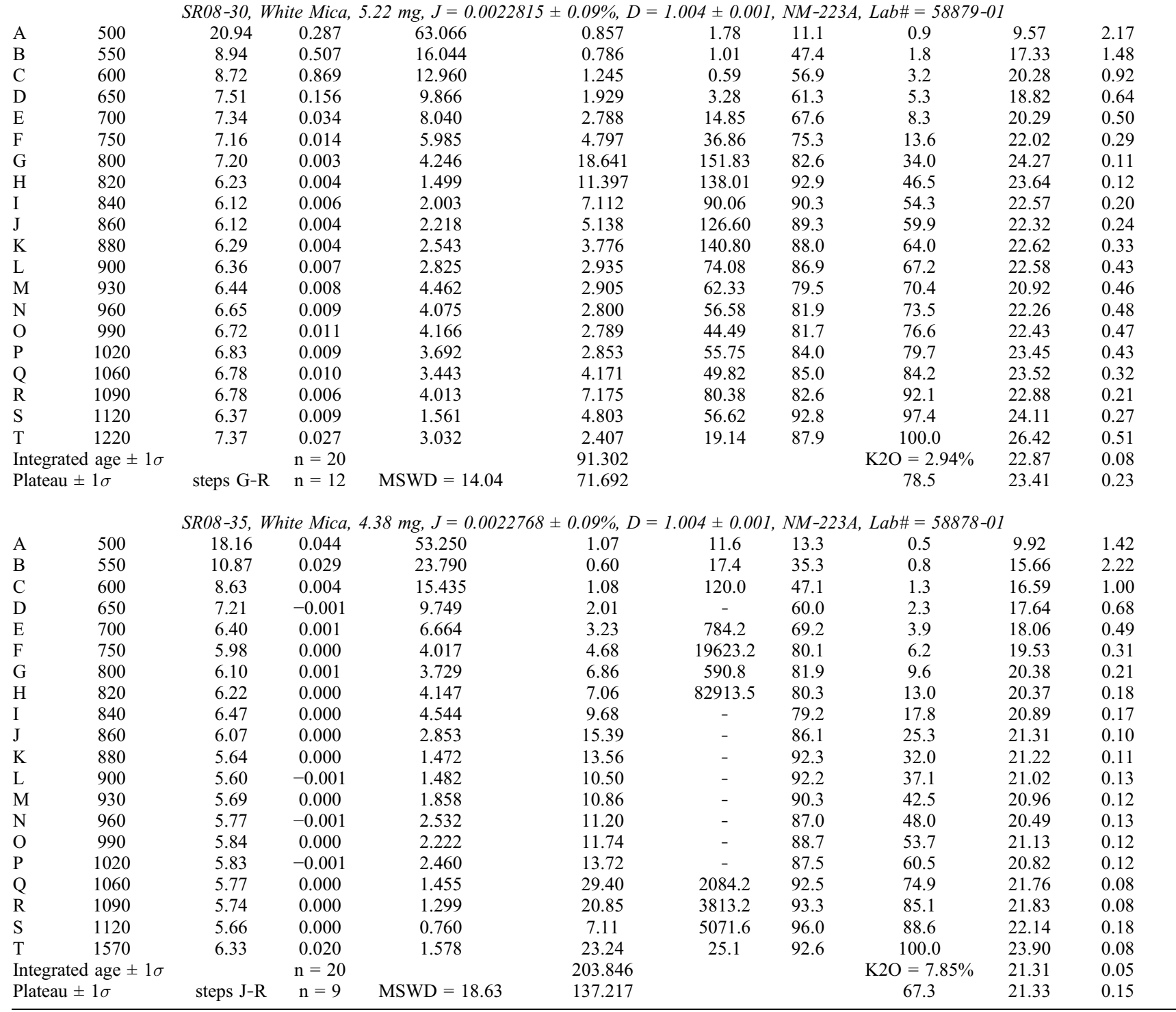


Table 1b. Summary of ${ }^{40} \mathrm{Ar} /{ }^{39} \mathrm{Ar}$ Results

\begin{tabular}{lccccccccc}
\hline Sample & L\# & Irrad & Mineral & Analysis & $\mathrm{n}$ & ${ }^{39}$ Ar $(\%)$ & MSWD & Plateau Age, Age (Ma) $\pm 1 \sigma$ & Integrated Age, ${ }^{\mathrm{a}}$ Age $(\mathrm{Ma}) \pm 1 \sigma$ \\
\hline SR08-4 & $58883-01$ & NM-223B & White Mica & Plateau & 7 & 53.1 & 39.7 & $26.95 \pm 0.21$ & $26.81 \pm 0.05$ \\
SR08-8 & $58882-01$ & NM-223B & White Mica & Plateau & 9 & 69.6 & 162.3 & $25.40 \pm 0.39$ & $25.39 \pm 0.05$ \\
SR08-11A & $58881-01$ & NM-223A & White Mica & Plateau & 9 & 64.0 & 66.6 & $24.49 \pm 0.27$ & $23.98 \pm 0.05$ \\
SR08-23 & $58880-01$ & NM-223A & White Mica & Plateau & 11 & 77.0 & 21.4 & $23.01 \pm 0.15$ & $22.91 \pm 0.05$ \\
SR08-30 & $58879-01$ & NM-223A & White Mica & Plateau & 12 & 78.5 & 14.0 & $23.41 \pm 0.23$ & $22.87 \pm 0.08$ \\
SR08-35 & 58878-01 & NM-223A & White Mica & Plateau & 9 & 67.3 & 18.6 & $21.33 \pm 0.15$ & $21.31 \pm 0.05$ \\
\hline
\end{tabular}

${ }^{\mathrm{a}}$ Integrated age means total gas age.

may explain the geometry of lenticular mica fish [Lister and Snoke, 1984] if rotation of cleavage planes toward the shear plane initiates a recrystallization process involving inward motion of recrystallization fronts and the migration of subgrain boundaries in muscovite fish. Muscovite growth parallel to the (001) plane gives rise to the observed asymmetric microstructure. Lister and Snoke [1984] note that such a process results in replacement of the deformed mica clast without changing its external shape and does not require changes in composition or detectable zoning pattern. No significant difference in composition of muscovite cores, rims, and tips is observed in muscovite samples collected in the top $83 \mathrm{~m}$ of the NSRD footwall mylonite (muscovite composition in auxiliary material). ${ }^{1}$

[20] Recrystallization involving solution-precipitation [Wilson and Bell, 1979; Dunlap, 1992; Mulch et al., 2005] could also explain the formation of mica fish belonging to group 1, group 2 and/or the neocrystallization of muscovite in strain shadows. This mechanism requires dissolution and mass transport via a fluid phase and precipitation at low pressure sites in the mylonite. The predominance of muscovite fish with their (001) planes subparallel to elongate quartz grains (oblique to the shear plane) can be explained by growth of mica in this direction (close to the instantaneous elongation direction); fluids that are saturated in nutrients from mica dissolution facilitate precipitation and growth of new mica. The very fine-grained muscovite tails extending from the mica fish likely grew also from dissolved muscovite porphyroclasts. This mechanism involving solution-precipitation is supported by the absence of intragrain deformation features (e.g., kink bands, micro fractures) in muscovite cores and tips.

[21] Muscovite microstructures are distinctly different in deeper parts $(>178 \mathrm{~m})$ of the Hendry's Creek section (Figures 6d, 6e, and 6f). Muscovite grains are thinner and elongate in the lineation direction; they lack the asymmetric sigmoidal shape, and are generally connected by foliationparallel tails that do not show stair stepping. Their cleavage planes are parallel to the mylonitic foliation or at a small synthetic angle. According to ten Grotenhuis et al. [2003], these mica grains result from synthetic slip on (001) planes. The lack of pressure shadows or trails of fine mica extending from the tips suggest that this muscovite may be part of the Cretaceous metamorphic assemblage and did not undergo significant recrystallization during mylonitic deformation in the NSRD footwall. This is also supported by microprobe data, which show distinct com-

\footnotetext{
${ }^{1}$ Auxiliary materials are available in the HTML. doi:10.1029/ 2010 TC002797.
}

positions at different depth intervals of the mylonitic footwall (auxiliary material).

\subsection{The ${ }^{40} \mathrm{Ar} /{ }^{39} \mathrm{Ar}$ Results}

[22] We analyzed six samples of muscovite from mylonitic quartzite uniformly distributed over ca. $300 \mathrm{~m}$ of footwall mylonite (Figure 6; Tables 1a and $1 \mathrm{~b}$ ) by furnace step heating ${ }^{40} \mathrm{Ar} /{ }^{39} \mathrm{Ar}$ geochronology (analytical procedures in auxiliary material; Table 2). Multigrain muscovite separates from samples collected over the top $83 \mathrm{~m}$ of the footwall mylonite have disturbed age spectra that show a monotonous rise in apparent age during the low-temperature release steps followed by a "saddle" shaped release spectrum at higher temperatures. Plateau ages calculated for these samples include $>50 \%$ of total ${ }^{39} \mathrm{Ar}$ released in at least seven successive steps. Apparent ${ }^{40} \mathrm{Ar} /{ }^{39} \mathrm{Ar}$ ages of muscovite fish are $26.9 \pm 0.2 \mathrm{Ma}$ (SR08-4; at $15 \mathrm{~m}$ from the NSRD), $25.4 \pm 0.4 \mathrm{Ma}$ (SR08-8; $55 \mathrm{~m}$ ), and $24.5 \pm 0.3 \mathrm{Ma}$ (SR08-11A; $75 \mathrm{~m})$. These plateau ages are consistent with total gas ages (TGA), which are $26.81 \pm 0.05 \mathrm{Ma}, 25.39 \pm$ $0.05 \mathrm{Ma}$ and $23.98 \pm 0.05 \mathrm{Ma}$, respectively.

$[23]{ }^{40} \mathrm{Ar} /{ }^{39} \mathrm{Ar}$ spectra from samples collected below $83 \mathrm{~m}$ become progressively flat with increasing distance beneath the NSRD. SR08-23 (178 m) and SR08-30 $(226 \mathrm{~m})$ provide plateau ages of $23.0 \pm 0.2 \mathrm{Ma}$ and $23.4 \pm 0.2 \mathrm{Ma}$, respectively, that include $>75 \%$ of ${ }^{39} \mathrm{Ar}$ released. Sample SR0835 at the base of the section $(275 \mathrm{~m})$ provides the youngest plateau age at $21.3 \pm 0.2 \mathrm{Ma}$ for $67.3 \%$ of ${ }^{39} \mathrm{Ar}$ released. Although all samples display some degree of disturbance, there is a general trend to more coherent release spectra and increasingly younger ${ }^{40} \mathrm{Ar} /{ }^{39} \mathrm{Ar}$ ages with increasing distance to the NSRD; a result consistent with the data acquired by Lee and Sutter [1991].

\section{Stable Isotope Geochemistry}

\subsection{Hydrogen Isotope Composition of Muscovite}

[24] The hydrogen isotope composition of muscovite was analyzed in 34 samples of mylonitic quartzite and schist collected over $c a .300 \mathrm{~m}$ of section beneath the NSRD (Figure 7 and Table 2; analytical procedures in Appendix B). Muscovite shows $\delta$ D values of about $-150 \%$ $( \pm 3.8 \%$; all values with respect to SMOW) at the top of the section and attains progressively higher values of up to $-64 \%$ toward the base of the section. This general trend is disrupted at two depth intervals (15 to $25 \mathrm{~m}$ and 91 to $144 \mathrm{~m}$ ) where muscovite $\delta \mathrm{D}$ increases rapidly to relatively high values $(-76 \%$ and $-63 \%$, respectively). Below the compositionally distinct $91-144 \mathrm{~m}$ interval, medium- to coarse-grained quartzite ( $\sim 50 \mathrm{~m}$ thick) contains muscovite 
Table 2. Hydrogen Isotope Data, Footwall Mylonite of the Northern Snake Range Detachment, Nevada

\begin{tabular}{|c|c|c|c|c|c|}
\hline Sample & $\begin{array}{c}\mathrm{dDms} \\
\text { (per mil) }\end{array}$ & $\begin{array}{l}\text { Fraction } \\
\text { Size }(\mu \mathrm{m})\end{array}$ & $\begin{array}{c}\text { Distance to } \\
\text { Hanging Wall (m) }\end{array}$ & $\begin{array}{l}\text { Universal Transvers } \\
\text { Coordinates (WGS84 }\end{array}$ & $\begin{array}{l}\text { Mercator } \\
\text { Projection) }\end{array}$ \\
\hline SR08-3 & -145 & $100<\mathrm{f}<180$ & 10 & $11 \mathrm{~S} 0752452$ & 4344296 \\
\hline SR08-4 & -76 & $100<\mathrm{f}<180$ & 15 & $11 \mathrm{~S} 0752389$ & 4344323 \\
\hline SR08-5A & -84 & $100<\mathrm{f}<180$ & 25 & $11 \mathrm{~S} 0752357$ & 4344330 \\
\hline SR08-5B & -92 & $100<\mathrm{f}<180$ & 25 & $11 \mathrm{~S} 0752357$ & 4344330 \\
\hline SR08-6A & -148 & $100<\mathrm{f}<180$ & 35 & $11 \mathrm{~S} 0752334$ & 4344311 \\
\hline SR08-6A & -150 & $180<\mathrm{f}<250$ & 35 & $11 \mathrm{~S} 0752334$ & 4344311 \\
\hline SR08-6B & -122 & $180<\mathrm{f}<250$ & 35 & $11 \mathrm{~S} 0752334$ & 4344311 \\
\hline SR08-7 & -144 & $180<\mathrm{f}<250$ & 45 & $11 \mathrm{~S} 0752335$ & 4344289 \\
\hline SR08-7 & -144 & $100<\mathrm{f}<180$ & 45 & $11 \mathrm{~S} 0752335$ & 4344289 \\
\hline SR08-8 & -139 & $100<\mathrm{f}<180$ & 55 & $11 \mathrm{~S} 0752338$ & 4344201 \\
\hline SR08-9 & -140 & $100<\mathrm{f}<180$ & 60 & $11 \mathrm{~S} 0752395$ & 4344117 \\
\hline SR08-10 & -143 & $100<\mathrm{f}<180$ & 65 & $11 \mathrm{~S} 0752352$ & 4344153 \\
\hline SR08-11A & -149 & $180<\mathrm{f}<250$ & 75 & $11 \mathrm{~S} 0752343$ & 4344139 \\
\hline SR08-12 & -145 & $100<\mathrm{f}<180$ & 83 & $11 \mathrm{~S} 0752361$ & 4344111 \\
\hline SR08-13 & -92 & $100<\mathrm{f}<180$ & 91 & $11 \mathrm{~S} 0752340$ & 4344116 \\
\hline SR08-14A & -90 & $100<\mathrm{f}<180$ & 97 & $11 \mathrm{~S} 0752354$ & 4344088 \\
\hline SR08-16 & -63 & $100<\mathrm{f}<180$ & 110 & 11S0750896 & 4345146 \\
\hline SR08-17A & -91 & $100<\mathrm{f}<180$ & 115 & $11 \mathrm{~S} 0750881$ & 4345115 \\
\hline SR08-18A & -82 & $100<\mathrm{f}<180$ & 130 & $11 \mathrm{~S} 0750859$ & 4345073 \\
\hline SR08-19 & -81 & $100<\mathrm{f}<180$ & 134 & $11 \mathrm{~S} 0750855$ & 4345062 \\
\hline SR08-20 & -92 & $100<\mathrm{f}<180$ & 144 & $11 \mathrm{~S} 0750855$ & 4345062 \\
\hline SR08-21 & -113 & $100<\mathrm{f}<180$ & 158 & 11S0750877 & 4345033 \\
\hline SR08-21 & -112 & $180<\mathrm{f}<250$ & 158 & 11S0750886 & 4345026 \\
\hline SR08-22 & -108 & $100<\mathrm{f}<180$ & 168 & 11S0750911 & 4345026 \\
\hline SR08-23 & -126 & $180<\mathrm{f}<250$ & 178 & 11S0750911 & 4345006 \\
\hline SR08-24 & -117 & $100<\mathrm{f}<180$ & 188 & & \\
\hline SR08-24 & -114 & $180<\mathrm{f}<250$ & 188 & & \\
\hline SR08-25 & -82 & $180<\mathrm{f}<250$ & 198 & & \\
\hline SR08-26 & -75 & $100<\mathrm{f}<180$ & 200 & & \\
\hline SR08-27 & -76 & $180<\mathrm{f}<250$ & 208 & & \\
\hline SR08-28 & -73 & $100<\mathrm{f}<180$ & 216 & $11 \mathrm{~S} 0750849$ & 4344963 \\
\hline SR08-29 & -73 & $100<\mathrm{f}<180$ & 223 & & \\
\hline SR08-30 & -78 & $100<\mathrm{f}<180$ & 226 & 11S0750878 & 4344938 \\
\hline SR08-31 & -88 & $100<\mathrm{f}<180$ & 236 & $11 \mathrm{~S} 0750903$ & 4344926 \\
\hline SR08-32 & -64 & $100<\mathrm{f}<180$ & 246 & $11 \mathrm{~S} 0750935$ & 4344893 \\
\hline SR08-33 & -74 & $100<\mathrm{f}<180$ & 255 & & \\
\hline SR08-34 & -72 & $100<\mathrm{f}<180$ & 265 & & \\
\hline SR08-34 & -72 & $180<\mathrm{f}<250$ & 265 & & \\
\hline SR08-35 & -77 & $100<\mathrm{f}<180$ & 275 & & \\
\hline
\end{tabular}

with $\delta \mathrm{D}$ values of ca. -108 to $-126 \%$. The base of the section is characterized by high muscovite $\delta \mathrm{D}$ values, -64 to $-72 \%$, which are typical for $\delta \mathrm{D}$ values in metamorphic rocks.

\subsection{Oxygen Isotope Thermometry of Mylonitic Quartzite}

[25] In addition to the hydrogen stable isotope data, $\delta^{18} \mathrm{O}$ values of quartz $\left(\delta^{18} \mathrm{O}_{\mathrm{qtz}}\right)$ and muscovite $\left(\delta^{18} \mathrm{O}_{\mathrm{ms}}\right)$ pairs were measured for oxygen isotope exchange thermometry (8 samples; Table 3 and Figure 7). While absolute temperatures strongly depend on the choice of calibration, oxygen isotope thermometry has the potential to determine relative temperature differences within rapidly cooled rocks with a precision of about $\pm 25^{\circ} \mathrm{C}$. The $\delta^{18} \mathrm{O}$ values in the detachment footwall range from $+11.4 \%$ to $+13.8 \%$ (quartz) and $+8.2 \%$ to $+11.5 \%$ (muscovite). Mylonite samples collected in the top $188 \mathrm{~m}$ of section tend to have lower $\delta^{18} \mathrm{O}_{\mathrm{qtz}}$ and $\delta^{18} \mathrm{O}_{\mathrm{ms}}$ values compared to their deeper counterparts, and temperature-dependent quartz-muscovite oxygen isotope fractionation $\left(\Delta^{18} \mathrm{O}_{\mathrm{qtz}-\mathrm{ms}}\right)$ falls within a range of 2.9 to $3.7 \%$ (excluding SR08-6A) in that section. In contrast, values for $\Delta^{18} \mathrm{O}_{\mathrm{qtz}-\mathrm{ms}}$ at the base of the section range from 1.8 to $2.9 \%$, with significant scatter in the data, precluding any direct temperature estimates.
[26] Taken together, $\delta^{18} \mathrm{O}$ and $\delta \mathrm{D}$ values are consistent with recrystallization and oxygen isotope exchange of quartz and mica with meteoric water and higher time-integrated water-rock ratios within the top $188 \mathrm{~m}$ of the section (Figure 7 and Table 2). Assuming oxygen isotope equilibrium during deformation and recrystallization in the top $188 \mathrm{~m}$ of the detachment footwall, $\Delta^{18} \mathrm{O}_{\mathrm{qtz}-\mathrm{ms}}$ values of 2.9 to $3.7 \%$ are consistent with deformation temperatures in the range of $402 \pm 52^{\circ} \mathrm{C}$ (using the calibration of Chacko et al. [1996] and samples SR08-3, SR08-4, SR08-12, SR0821 and SR08-24; Table 3), which are slightly higher than those (ca. $325^{\circ} \mathrm{C}$ ) inferred by Lee and Sutter [1991] on the basis of ${ }^{40} \mathrm{Ar} /{ }^{39} \mathrm{Ar}$ closure temperatures of muscovite and microcline from footwall rocks of the NSRD.

[27] In contrast, samples collected below $188 \mathrm{~m}$ have significantly lower quartz-muscovite fractionation values $\left(\Delta^{18} \mathrm{O}_{\mathrm{qtz}-\mathrm{ms}} \approx 1.8\right.$ to 2.9$)$ suggesting oxygen isotope exchange at higher $\left(500-600^{\circ} \mathrm{C}\right)$ temperatures. However, the relatively poor reproducibility of measured $\delta^{18} \mathrm{O}$ values (SR08-26 and SR08-34, Table 3) and the presence of large quartz grain relics and newly recrystallized grains (Figure 4) indicates lack of sample-scale recrystallization and most likely lack of oxygen isotope equilibrium during deformation. Using oxygen isotope exchange temperatures of $402 \pm$ 


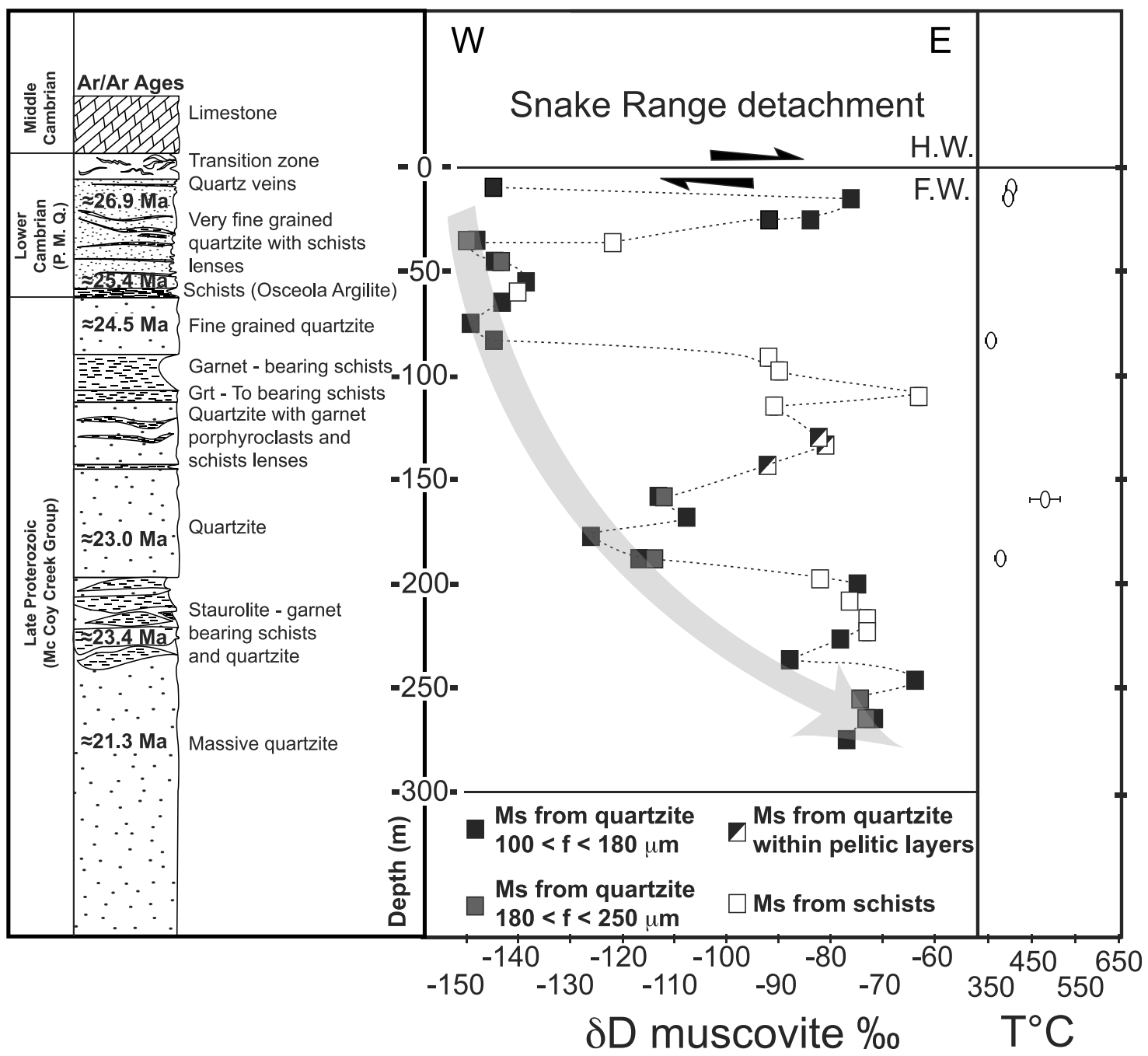

Figure 7. The $\delta \mathrm{D}$ values of muscovite from mylonitic quartzite in the footwall of the Northern Snake Range Detachment. (left) Lithologic column that can be correlated with samples from which $\delta \mathrm{D}$ values are obtained. (right) Oxygen isotope exchange temperatures based on quartz-muscovite fractionation. HW: hanging wall, FW: footwall.

$52^{\circ} \mathrm{C}$, the hydrogen isotope muscovite-water fractionation of Suzuoki and Epstein [1976] and measured $\delta \mathrm{D}_{\mathrm{ms}}$ values of samples within the top $83 \mathrm{~m}$ of the detachment footwall, water percolating at the detachment had $\delta \mathrm{D}_{\text {water }}$ values of $\leq-115 \pm 5 \%$.

[28] Equilibrium oxygen isotope quartz-muscovite exchange within the top $188 \mathrm{~m}$ of footwall mylonite at temperatures of $402 \pm 52^{\circ} \mathrm{C}$ implies that quartz and muscovite share a common recrystallization history. This equilibrium is consistent with observed quartz microstructures displaying a homogeneously recrystallized quartz matrix and suggests that the recrystallization of pre-mylonitic muscovites occurred in the presence of meteoric fluids at the same time as a) recrystallization of the quartz matrix and b) newly formed micas producing shear bands (Figure 6).

[29] Samples collected below $188 \mathrm{~m}$ did not attain syndeformational oxygen isotope equilibrium owing to the preservation of Mesozoic mineral assemblages (quartzplagioclase-muscovite-biotite \pm garnet \pm staurolite) and quartz microstructures (mixing of large quartz grain relics and newly recrystallized grains), which explains the poor reproducibility of temperature-dependent oxygen isotope fractionation at the base of the section (Table 3).

\section{Discussion}

[30] Detachments like the NSRD that separate brittle upper crust from ductile footwall rocks are commonly underlain by mylonite, $10 \mathrm{~s}$ to $100 \mathrm{~s}$ of meters thick, that accommodate large amounts of extensional strain and act as a deforming interface for metamorphic fluids at depth and meteoric fluids that permeate the brittle crust and circulate within and below the detachment. Deciphering the spatial pattern of fluid migration and its relationship to strain localization processes and assessing the isotopic composition, and therefore the origin of fluids within extensional shear zones, provide invaluable information on crustalscale fluid flow and the thermal and mechanical implications of large-scale extension [e.g., Mulch et al. 2006; Gottardi et al., 2011]. These studies also inform about the paleo- 
Table 3. Oxygen Isotope Data and Associated Oxygen Isotope Exchange Temperatures Using the Calibration of Chacko et al. $[1996]^{\mathrm{a}}$

\begin{tabular}{cccccc}
\hline Sample & $\begin{array}{c}\delta^{180} \text { qtz, } \\
\text { (per mil) }\end{array}$ & $\begin{array}{c}\delta^{180} \mathrm{~ms}, \\
(\text { per mil) }\end{array}$ & $\Delta$ qtz-ms & $\begin{array}{c}\text { Temperature } \\
\left({ }^{\circ} \mathrm{C}\right)\end{array}$ & $\begin{array}{c}\text { Distance } \\
\text { to Hanging } \\
\text { Wall }(\mathrm{m})\end{array}$ \\
\hline SR08-3 & 11.40 & 8.48 & 2.92 & 419 & 10 \\
SR08-3 & 11.37 & 8.24 & 3.13 & 396 & \\
& & & & & \\
SR08-4 & 11.96 & 8.79 & 3.17 & 393 & 15 \\
SR08-4 & 12.02 & 8.87 & 3.15 & 393 & \\
& & & & & \\
SR08-6A & 11.45 & 9.60 & 1.85 & 593 & 35 \\
SR08-6A & 11.58 & 9.99 & 1.59 & 662 & \\
& & & & & \\
SR08-12 & 12.89 & 9.42 & 3.47 & 362 & 83 \\
SR08-12 & 13.42 & 9.74 & 3.68 & 344 & \\
& & & & & \\
SR08-21 & 12.41 & 10.26 & 2.15 & 533 & 158 \\
SR08-21 & 12.68 & 9.86 & 2.82 & 431 & \\
& & & & & \\
SR08-24 & 12.71 & 9.28 & 3.43 & 366 & 200 \\
SR08-24 & 12.56 & 9.35 & 3.21 & 388 & \\
& & & & & \\
SR08-26 & 13.83 & 10.98 & 2.85 & 428 & 265 \\
SR08-26 & 13.38 & 11.48 & 1.90 & 583 & \\
SR08-26 & 13.25 & 11.39 & 1.86 & 592 & \\
& & & & & \\
SR08-34 & 12.47 & 10.12 & 2.35 & 498 & \\
SR08-34 & 11.85 & 10.08 & 1.77 & 611 & \\
\hline
\end{tabular}

${ }^{\mathrm{a}}$ The two or three measurements per sample represent duplicates.

topography of the region undergoing extension [e.g., Mulch et al. 2007; Mulch and Chamberlain, 2007] since the hydraulic head generated in high-relief areas may be an important driving force for hydrothermal fluid circulation in detachment systems [Person et al., 2007]. Meteoric fluids, therefore, provide a critical link between processes that characterize the internal dynamics of orogens and those that shape the Earth's surface.

[31] Here, we focus on meteoric water that attained depths corresponding to the brittle-ductile transition in quartzite during ductile deformation in NSRD footwall mylonite. Three lines of evidence lead us to conclude that muscovite recrystallized during mylonitization in the upper part of the mylonitic footwall. First, muscovitic white mica shows a systematic hydrogen isotope pattern that is consistent with a mixing relationship between meteoric and metamorphic water as a function of depth. The meteoric imprint dominates the top $188 \mathrm{~m}$ of mylonite, while the section below carries the signature of metamorphic fluids. Second, elemental compositions (see auxiliary material) reveal distinct muscovite populations at different depths in the footwall mylonite. Third, oxygen isotope ratios in muscovite are shifted toward lower values at the top of the footwall mylonite, again providing strong evidence for water-rock interaction with low $-\delta^{18} \mathrm{O}$ (and low- $\left.\delta \mathrm{D}\right)$ meteoric fluids.

[32] Collectively, all three compositional tracers require elemental and isotopic $\left(\mathrm{D} / \mathrm{H},{ }^{18} \mathrm{O} /{ }^{16} \mathrm{O}\right)$ exchange at the lattice scale even for major elements, which in turn renders it highly unlikely that the intragrain radiogenic ${ }^{40} \mathrm{Ar}$ distribution remained unaffected during mylonitic deformation. Therefore, based on combined microstructural, compositional, and isotopic evidence, we interpret the ${ }^{40} \mathrm{Ar} /{ }^{39} \mathrm{Ar}$ ages from those intervals of the footwall mylonite that display clear-cut evidence for grain-scale syn-mylonitic hydrogen and oxygen isotope exchange $(0-83 \mathrm{~m}$ and given the low $\delta \mathrm{D}$ values potentially down to $188 \mathrm{~m}$ ) to reflect the timing of muscovite recrystallization, rather than cooling through closure to ${ }^{40} \mathrm{Ar}$ diffusion.

[33] The hydrogen and argon muscovite data are therefore tracking changes in fluid composition as well as the timing and duration of isotopic exchange during the transition from crustal-scale extension by (fluid-assisted?) ductile flow in the middle crust to an extensional system that is largely characterized by brittle normal faulting in the upper crust and footwall exhumation through tectonic (and erosional) unloading. Four main observations characterize the NSRD footwall at Hendry's Creek: 1) white mica from the top $83 \mathrm{~m}$ of the section is recrystallized and dynamic quartz - muscovite microstructures define the very strong Oligo-/Miocene lineation and foliation; 2) ${ }^{40} \mathrm{Ar} /{ }^{39} \mathrm{Ar}$ geochronology yields Oligo-/Miocene muscovite ages that decrease systematically with depth in the detachment; 3) quartz-muscovite oxygen isotope equilibrium fractionation $\left(\Delta^{18} \mathrm{O}_{\mathrm{qtz}-\mathrm{ms}} \approx 2.9-3.7 \%\right.$ o in the upper part $(0-188 \mathrm{~m})$ of the detachment indicates deformation temperatures of $402 \pm 52^{\circ} \mathrm{C}$, whereas the quartz-muscovite oxygen isotope fractionation at the base of the detachment $(200-275 \mathrm{~m})$ is much smaller $\left(\Delta^{18} \mathrm{O}_{\mathrm{qtz}-\mathrm{ms}} \approx 1.8-2.9 \%\right.$ ) and subject to large intrasample variability suggesting lack of sample-scale oxygen isotope equilibrium; 4) $\delta \mathrm{D}_{\text {muscovite }}$ values as low as $-150 \%$ require the presence of meteoric fluids during deformation in the detachment footwall. However, $\delta \mathrm{D}$ values increase progressively toward metamorphic values at the base of the detachment, with systematic deviations from this trend in two layers that were preserved from significant time-integrated exchange with meteoric fluids.

\subsection{Meteoric Fluid Migration in the NSRD Footwall Mylonite}

[34] The muscovite hydrogen (and to some extent oxygen) isotope compositions collected within the top $188 \mathrm{~m}$ of the NSRD mylonitic footwall indicate the presence of meteoric water during deformation and muscovite recrystallization at $\mathrm{T}=402 \pm 52^{\circ} \mathrm{C}$ in the ductile segment of the NSRD. These results extend the study of Losh [1997] who described the presence of meteoric water in upper-plate faults, fault breccia, and lower plate mylonite. Oxygen isotope data of stylolitic calcite breccia display large variability ranging from $-1.6 \%$ to $+15.3 \%$ o that reflect meteoric fluid-rock interaction at varying temperatures $\left(\mathrm{T}=120-300^{\circ} \mathrm{C}[\right.$ Losh, 1997]). Similarly, calcite from upper plate faults displays a wide range of $\delta^{18} \mathrm{O}$ values between $-2.4 \%$ and $+16.3 \%$, both data sets being consistent with the infiltration of meteoric water in the upper plate having $\delta^{18} \mathrm{O}_{\text {water }} \leq-12 \%$ and $\delta \mathrm{D}_{\text {water }} \leq-86 \%$ [Losh, 1997]. Structurally below 188 $\mathrm{m}$, our data set shows no further evidence for such meteoric fluids. The close correlation of $\delta \mathrm{D}$ values, structural position, and lithological changes across the exposed section of the NSRD footwall permits two alternative explanations for the $\delta \mathrm{D}$ data: 1$)$ the time-integrated infiltration of meteoric fluids diminished down section or alternatively 2) the hydrogen isotopic composition of meteoric water at the source became less negative through time, as suggested by the younging of ${ }^{40} \mathrm{Ar} r^{\beta 9} \mathrm{Ar}$ ages downward. 
[35] We consider it unlikely that the increasing $\delta \mathrm{D}$ values with depth directly track the isotopic composition of meteoric fluids over time from 27 to 21 Ma: Compiled oxygen isotope in precipitation data for the Cenozoic western United States document that similar-to-modern rainfall and isotope in precipitation patterns developed as early as $40 \mathrm{Ma}$ and that low $-\delta \mathrm{D}$ values have characterized the central Great Basin since that time [Mix et al., 2011]. An increase in $\delta \mathrm{D}_{\text {water }}$ of ca. $70 \%$ as recorded across the NSRD footwall is strikingly at odds with any other surface proxy. Second, changes in climate or topography required to produce such an increase in $\delta \mathrm{D}_{\text {water }}$ at the Earth's surface [Poage and Chamberlain, 2001; Rowley and Currie, 2006; Rowley and Garzione, 2007] are far too large to be explained by the geologic record. Therefore, it appears most likely that a mixing relationship between meteoric and metamorphic fluids, or a decreased time-integrated influx of meteoric fluids downward explains the observed $\delta \mathrm{D}$-depth pattern. This requires the influx of low- $\delta \mathrm{D}$ meteoric waters $(\delta \mathrm{D} \leq-115 \%)$ during several million years within the top $188 \mathrm{~m}$ of the NSRD footwall, which overprinted the hydrogen isotopic composition of metamorphic mica.

[36] The NSRD and associated footwall mylonite expose a complete cross section through a fossil syn-extensional hydrothermal system and permit the evaluation of flow paths in extensional systems. Because $\delta \mathrm{D}$ and $\delta^{18} \mathrm{O}$ values of pristine metamorphic silicate minerals are high compared to those of meteoric waters, any isotopic mineral-water interaction along the flow path of the infiltrating fluid would generally shift $\delta \mathrm{D}_{\text {water }}$ and $\delta^{18} \mathrm{O}_{\text {water }}$ to more positive values. The downsection increase in $\delta \mathrm{D}_{\text {muscovite }}$ values is therefore most readily explained in terms of decreasing time-integrated meteoric water-rock ratios with depth. Deviations from a simple attenuation pattern (arrow in Figure 7) exist between 91 and $144 \mathrm{~m}$ and between 200 and $250 \mathrm{~m}$ (Figure 7). In both cases white mica separates from schist units (or thin quartzite layers embedded in schist and pelite) show relatively high $\delta \mathrm{D}_{\text {muscovite }}$ values attaining values close to the metamorphic end-member of -60 to $-70 \%$.

[37] Hydrogen is a trace element in silicate rocks with concentrations on the order of 2000 ppm [e.g., Sheppard, 1986 ] and less than $500 \mathrm{ppm}$ in quartzite. Any change in modal abundance of hydrous phases (such as muscovite and biotite) therefore affects the potential hydrogen reservoir in deforming rocks. Following simple mass balance, we expect higher $\delta \mathrm{D}_{\text {mineral }}$ values in lithologies that are rich in hydrous minerals (schist) compared to their more mica-poor lithologies (quartzite). We interpret the high $\delta \mathrm{D}_{\text {muscovite }}$ values at 91-144 $\mathrm{m}$ and $200-250 \mathrm{~m}$ to be lithologically controlled owing to the presence of garnet-bearing mica and staurolitegarnet schist, respectively, as the dominant rock types. We conclude that lithological differences (modal abundance of hydrous silicate phases) within the NSRD footwall and along the flow path significantly affected $\delta \mathrm{D}_{\text {water }}$ and ultimately $\delta \mathrm{D}_{\text {muscovite }}$ values during deformation.

[38] In our view, the NSRD represents a critical interface for syn-extensional meteoric fluid flow that attained depths corresponding to the brittle-ductile transition. At any given time during deformation meteoric fluids were present in the structurally highest compartments of the footwall mylonite, implying that meteoric fluids entered the ductile footwall on the scale of $10 \mathrm{~s}$ of meters.

\subsection{Interpretation of ${ }^{40} \mathrm{Ar} /{ }^{39} \mathrm{Ar}$ Data From Mylonitic Quartzite}

[39] Two main observations characterize our ${ }^{40} \mathrm{Ar} /{ }^{39} \mathrm{Ar}$ data. First, apparent ages become increasingly younger with distance from the hanging wall (and the NSRD), and second, even though relatively young ages during the early release steps of the age spectrum persist, complex release spectra become increasingly plateau-like toward the base of the section. Similar observations have been presented by Lee and Sutter [1991] and this apparent ${ }^{40} \mathrm{Ar} /{ }^{39} \mathrm{Ar}$ age trend has been interpreted to reflect increasing temperatures with depth during ductile extension where samples closest to the hanging wall were not maintained at sufficiently high temperature to achieve complete degassing and resetting of Cretaceous micas after $28 \mathrm{Ma}$.

[40] We generally concur with the results of Lee and Sutter [1991]; yet we caution that the assumptions of the closure temperature concept are frequently compromised for rapidly exhuming and deforming/recrystallizing rocks (e.g., changes in grain size, cooling rate, mineral composition [e.g., Mulch and Cosca, 2004; Mulch et al., 2005]. In particular, we suggest that the change in the shape of ${ }^{40} \mathrm{Ar} /{ }^{39} \mathrm{Ar}$ release spectra and the decrease in age with structural depth may not simply be the result of differential cooling but of detachment-wide ductile deformation between 27 and 23 Ma recorded by recrystallization ages in the top ca. $188 \mathrm{~m}$ of the detachment. This interpretation is supported by muscovite that deformed and most likely recrystallized by combined dissolution/precipitation and migration recrystallization (asymmetric/sigmoidal mica fish, shear bands, development of muscovite tails) in a matrix of quartz that displays completely recrystallized neoblasts by subgrain rotation from large elongate quartz grains. Results of quartzmuscovite oxygen isotope exchange thermometry indicate isotopic equilibrium between both minerals at temperatures of $402 \pm 52^{\circ} \mathrm{C}$ in the top $188 \mathrm{~m}$ of the NSRD footwall. The persistence of oxygen isotope equilibrium preserved in quartzite mylonite further implies that quartz and muscovite in samples from the top $188 \mathrm{~m}$ of detachment share a common recrystallization history. The relative rates of ${ }^{40} \mathrm{Ar}$, hydrogen, and oxygen diffusion in white mica at such temperature are poorly known; however, it appears unlikely that in an overall cooling system the $\mathrm{D} / \mathrm{H}$ and ${ }^{18} \mathrm{O} /{ }^{16} \mathrm{O}$ ratios of white mica were reset through recrystallization without affecting the ${ }^{40} \mathrm{Ar} /{ }^{39} \mathrm{Ar}$ ages in white mica. Even though ${ }^{40} \mathrm{Ar}$ retention of white mica during deformation depends mostly on the mica recrystallization process (irrespective of the source or composition of the fluid as long as ${ }^{40} \mathrm{Ar}$ concentrations in the fluid are low), we consider hydrogen as a conservative tracer for syn-deformational changes in mineral composition and the low $\delta \mathrm{D}$ values as compositional evidence for lattice-scale recrystallization (and isotope exchange) in muscovite of the NSRD footwall [e.g., Mulch et al., 2005]. Based on the relatively fast cooling rates deduced for the Snake Range MCC $\left(10-55^{\circ} \mathrm{C} / \mathrm{Ma}\right.$ [Lee, 1995], and $49-67^{\circ} \mathrm{C} / \mathrm{km}$ [Losh, 1997]) and protracted east-directed extensional shearing through the brittle-ductile transition, ages of $26.9 \pm 0.2 \mathrm{Ma}, 25.4 \pm$ $0.4 \mathrm{Ma}$ and $24.5 \pm 0.3 \mathrm{Ma}$ within the top- $83 \mathrm{~m}$ of the NSRD footwall are interpreted to reflect the timing and propagation of mylonitization. Although the $\delta \mathrm{D}$ value of 
sample SR08-23 $\left({ }^{40} \mathrm{Ar} /{ }^{39} \mathrm{Ar}\right.$ plateau age of $\left.23.0 \pm 0.2 \mathrm{Ma}\right)$ located at $178 \mathrm{~m}$ is higher $(\delta \mathrm{D}=-126 \%)$ than $\delta \mathrm{D}$ values within the top $83 \mathrm{~m}$ of the detachment, its relatively low $\delta \mathrm{D}$ value still requires isotopic exchange with meteoric water and suggests that E-directed non-coaxial deformation and meteoric-hydrothermal circulation were most likely active at this depth interval until about 23 Ma.

[41] For samples SR08-30 and SR08-35 (base of the section; 226 to $275 \mathrm{~m}$ ), ${ }^{40} \mathrm{Ar} /{ }^{39} \mathrm{Ar}$ release spectra are almost flat, and plateau ages of $23.4 \pm 0.2 \mathrm{Ma}$ and $21.3 \pm 0.2 \mathrm{Ma}$ are significantly younger than in the top $188 \mathrm{~m}$ of section. In this part of the section, measured $\delta \mathrm{D}$ values are relatively high $(-78$ to $-77 \%$ ), precluding the presence of significant amounts of meteoric water during recrystallization of muscovite. Therefore, meteoric fluids no longer penetrated to this depth and the meteoric-hydrothermal system in the detachment footwall became dysfunctional at around $23 \mathrm{Ma}$. This does not rule out the possibility that meteoric fluids percolated the brittle part of the detachment system (NSRD and hanging wall) during later stages of deformation (until ca. $17 \mathrm{Ma}$ [Miller et al., 1999a]), but there are no traces of such fluids in the exhumed mylonites. Moreover, the low quartz-muscovite fractionation values $\left(\Delta^{18} \mathrm{O}_{\mathrm{qtz}-\mathrm{ms}} \approx 1.8\right.$ to 2.9) and poor reproducibility measured in these samples indicate that oxygen in white mica and quartz did not equilibrate completely.

[42] This disequilibrium is also reflected in microstructures displaying a large range of quartz grain sizes ( $<40 \mu \mathrm{m}$ to $>200 \mu \mathrm{m}$, Figure $4 \mathrm{~d}$ ) including Oligo-Miocene recrystallized neoblasts and pre-mylonitic (Cretaceous?) quartz grains. We therefore interpret the relatively flat ${ }^{39} \mathrm{Ar}$ release spectra at the base of the section to represent cooling through the closure to ${ }^{40} \mathrm{Ar}$ diffusion in muscovite between 23 and $21 \mathrm{Ma}$ rather than the timing of recrystallization. We note that the increasingly flat spectra at greater depth could equally be interpreted in terms of an increasingly homogeneous mica population. Such a scenario can only be tested by in situ ${ }^{40} \mathrm{Ar} /{ }^{39} \mathrm{Ar}$ geochronology, however, given the relatively large intrasample variability in both mica microstructure and $\delta^{18} \mathrm{O}$ values between 200 and $275 \mathrm{~m}$, we consider it unlikely that deformation-recrystallization processes promoted a homogeneous intragrain ${ }^{40} \mathrm{Ar}$ distribution in these samples.

[43] The saddle-shaped age spectra obtained from samples within the top $188 \mathrm{~m}$ of the detachment could point to the presence of excess ${ }^{40} \mathrm{Ar}$. Commonly, excess ${ }^{40} \mathrm{Ar}$ results from re-equilibration of the analyzed mineral with unsupported ${ }^{40} \mathrm{Ar}$ in the fluid that is present during deformation and recrystallization [Cumbest et al., 1994]. However, $\delta \mathrm{D}$ values within this structural interval of the detachment are low (ca. $-145 \%$ ) supporting the idea that these minerals underwent syn-mylonitic isotope exchange in the presence of meteoric water. Since surface-derived fluids most likely had little time to incorporate unsupported ${ }^{40} \mathrm{Ar}$ (i.e., ${ }^{40} \mathrm{Ar}$ that is not the result of in situ decay of ${ }^{40} \mathrm{~K}$ ) and were initially in equilibrium with atmospheric argon, they are the best candidates to meet the assumption of an initial ${ }^{40} \mathrm{Ar}{ }^{36} \mathrm{Ar}$ ratio of 295.5 , and hence are not likely sources for excess ${ }^{40} \mathrm{Ar}$ [Mulch et al., 2005]. It is thus suggested that the saddle-shaped spectra combined with a monotonic rise in age in the low temperature part of the age spectrum reflect the combined effects of synkinematic white mica growth, cooling-related closure to argon diffusion, and potentially mixing of different white mica populations due to various degrees of recrystallization in the respective samples [Wijbrans and McDougall, 1986; Kirschner et al., 1996]. Nevertheless, we consider the systematic decrease in age in the footwall mylonite to be significant and to reflect a protracted history of recrystallization, deformation, and fluid flow in the detachment mylonites that lasted at least from ca. $27 \mathrm{Ma}$ to $23 \mathrm{Ma}$.

\subsection{Fluid Flow in Exhuming Detachment Systems}

[44] The combined $\delta \mathrm{D}, \delta^{18} \mathrm{O}$, and ${ }^{40} \mathrm{Ar}{ }^{39} \mathrm{Ar}$ data in footwall mylonite of the NSRD are consistent with a model in which the brittle-ductile transition of the Northern Snake Range MCC operated under roughly the same temperatures $\left(\sim 400^{\circ} \mathrm{C}\right)$ through time. If the ${ }^{40} \mathrm{Ar}{ }^{39} \mathrm{Ar}$ ages within the top $188 \mathrm{~m}$ of the detachment indeed reflect recrystallization and ductile non-coaxial top-to-east shearing, hot footwall material was advected at relatively high rates leading to strongly compressed isotherms in the NSRD footwall while at the same time protracted normal faulting and denudation at the Earth's surface continuously exhumed parts of the footwall. In such a continuously exhuming system, shearing rocks in the detachment footwall cool owing to the combined effects of exhumation and hydrothermal activity [Gottardi et al., 2011]. As a result, ductile deformation microstructures freeze, and the deformation-recrystallization front moves downward. Recrystallization-driven argon, hydrogen, and oxygen isotope exchange effectively ceases (as long as post-mylonitic diffusional exchange is insignificant). Ultimately, the detachment footwall exposed today depicts the effect of a protracted deformation history of the NSRD and its footwall mylonite and reflects the downward propagation of deformation with time from 27 to $23 \mathrm{Ma}$ within the ca. $300 \mathrm{~m}$ of mylonite exposed at Hendry's Creek.

[45] We propose that the meteoric hydrothermal system changed abruptly at around $23 \mathrm{Ma}$, such that meteoric fluids no longer penetrated the footwall of the NSRD. The driving forces of convective fluid flow in such tectonic setting are the hydraulic head provided by topography as well as density changes of fluid owing to changes in salinity and temperature [Person et al., 2007]. The topographic evolution of the Snake Range MCC is largely unconstrained, but there is no reason to believe that major reductions in topography affecting the hydraulic head occurred over the time intervals of interest. Therefore, the more likely change that initiated the shutdown of the meteoric-hydrothermal system is a major modification in the deformation-controlled porosity and permeability structure of the entire detachment system and the hanging wall-footwall interface in particular. [46] The combined ${ }^{40} \mathrm{Ar} /{ }^{39} \mathrm{Ar}$ and stable isotope results document that an important phase of ductile deformation and fluid-rock interaction in the NSRD footwall occurred between 27 and $23 \mathrm{Ma}$. This is not necessarily at odds with crosscutting relationships and volcanic stratigraphy of upper plate rocks. Miller et al. [1999a] suggest that mylonites started forming at $35 \mathrm{Ma}$ and continued until final exhumation at $17 \mathrm{Ma}$, as recorded by apatite and zircon fission track data. The last main extensional event in the NSRD history seems to not have been associated with hydrothermal activity at depth; at least there are no detectable traces of it 
in the detachment. Thus, we suggest that at ca. $23 \mathrm{Ma}$, when the crustal-scale hydrothermal system ceased, the brittleductile transition at the hanging wall-footwall interface stopped to be an effective locus of rapid heat advection. Isotherms that were previously compressed started to relax at that time. Cessation of hydrothermal activity in the detachment system does not mean that extensional deformation came to an end but requires a change in deformation style that affected the dynamic porosity and permeability structure of the deforming NSRD footwall that is exposed today at Hendry's Creek, in which no ductile deformation occurred after $23 \mathrm{Ma}$. By $17 \mathrm{Ma}$, the kind of hydrothermal system we have documented at Hendry's Creek may have migrated further east, in the deeper part of the dipping detachment system, and was never brought up to the surface.

\section{Conclusions}

[47] Combined microstructural, stable isotopic, and geochronologic data indicate that the fossil hydrothermal system of the Northern Snake Range MCC has been exhumed by the combined effects of extensional detachment faulting and denudation. Meteoric fluids that penetrated the brittle upper crust became involved in large-scale convective flow down to significant depth $(5-15 \mathrm{~km})$ over the time scale of mylonite formation (ca. 27 to $23 \mathrm{Ma}$ ). Based on the absence of meteoric fluids in the deeper part of the exposed NSRD footwall, we suggest that ductile deformation and meteoric hydrothermal activity in the footwall of the NSRD shut down after $23 \mathrm{Ma}$. At the same time, isotherms that were strongly compressed in the NSRD footwall between 27 and 23 Ma due to the combined effects of upper crustal thinning and lower plate heat advection must have started to relax.

[48] We further suggest that at ca. $23 \mathrm{Ma}$, when the exhumed hydrothermal system ceased, the brittle-ductile interface at the transient hanging wall-footwall interface stopped to be an effective means of rapid heat advection and that subsequently extensional strain was localized through brittle deformation mechanisms. Together with results from metamorphic core complexes in the northern North American Cordillera (e.g., Shuswap and Kettle [Mulch et al., 2004, 2007]; Raft River [Gottardi et al., 2011]) microstructural and isotopic evidence summarized in this paper demonstrates that meteoric hydrothermal systems represent key features of extensional detachments that involve lateral and vertical mass transfer, denudation, and ultimately Cenozoic development of topography in the North American Cordillera [Mulch and Chamberlain, 2007; Mix et al., 2011].

\section{Appendix A: Electron Backscatter Diffraction Method and Analytical Procedure}

[49] Quartz preferred orientations were measured by scanning electron microscopy using electron backscatter diffraction (EBSD) [Lloyd et al., 1991; Adams et al., 1993; Dingley and Field, 1997] on the EBSD/SEM system at the University of Minnesota, CHARFAC facilities, Minneapolis, USA. Backscattered electron (BSE) diffraction on the scanning electron microscope has become an important tool for the combined study of microstructures in crystalline materials. It is possible to measure complete crystallographic orientations of single crystallites with direct reference to the microstructure [Neumann, 2000; Heidelbach et al., 2000]. EBSD is based on the automatic analysis of diffraction patterns. These patterns, composed of Kikuchi bands, are generated by the interaction of a vertical incident electron beam with a highly tilted, $70^{\circ}$, flat crystal surface. A phosphor screen is located close to the polished thin section to collect the diffraction pattern, which is then processed and indexed using the CHANNEL+ software [Schmidt and Olesen, 1989]. The precision of crystal orientations measured from electron backscattering patterns is better than $1^{\circ}$ [Krieger Lassen, 1996]. SEM conditions used were $25 \mathrm{~mm}$ working distance, $20 \mathrm{kV}$ accelerating voltage and $\sim 10 \mathrm{nA}$ beam current. For the EBSD analysis we used the following indexing parameters; 6-8 bands, band edges, high resolution of 70, 4 frames of noise reduction, 70 reflectors and a 1.2 MAD cutoff.

\section{Appendix B: Stable Isotope Analyses}

[50] The $\delta \mathrm{D}$ values of hydrous silicates were determined by continuous flow mass spectrometry using a high temperature elemental analyzer (Thermo Finnigan TC/EA) coupled to a mass spectrometer (Delta V Advantage). Three internationally referenced standard materials and additional in-house working standards were run with the samples. After correction of mass bias, daily drift of the thermal combustion reactor and offset from the certified reference values, NBS30 (biotite), NBS22 (oil), CH7 (polyethylene foil) had $\delta \mathrm{D}=-65.8 \%,-118.6 \%$, and $-102.1 \%$, respectively. Repeated measurements of various standards and unknowns gave a precision of $\pm 2 \%$ for $\delta \mathrm{D}$. All isotopic ratios are reported relative to standard mean ocean water (VSMOW).

[51] The $\delta^{18} \mathrm{O}$ values of quartz and muscovite pairs were measured using laser-fluorination mass spectrometry. About 1.2 to $1.5 \mathrm{mg}$ of muscovite and quartz were heated using a $\mathrm{CO}_{2}$-laser fluorination line coupled to a Finnigan MAT253 mass spectrometer in the Stable Isotope Laboratory at the University of Lausanne, Switzerland. Mineral pairs were measured within the same run together with at least two mineral standards. All samples were run in duplicate, and measurements were accepted if duplicate analyses were within $\pm 0.25 \%$. $\delta^{18} \mathrm{O}$ values are normalized to values of $9.6 \%$ measured for NBS-28 quartz and average precision of in-house standards is $\pm 0.1 \%$.

[52] Acknowledgments. This study was partially funded through a Leibniz University young investigator grant "Wege in die Forschung" to A.G. A.M. acknowledges support through DFG-INST 187/400-1 FUGG, U.S. NSF EAR-1019648, and the LOEWE funding program of Hesse's Ministry of Higher Education, Research, and the Arts. Part of this work was carried out in the Institute of Technology Characterization Facility, University of Minnesota, which receives partial support from NSF though the MRSEC program. C.T. acknowledges support from Swiss Science Foundation project FNS-117694 and U.S. NSF EAR-0838541. We thank A. Quilichini, B. Putlitz (Lausanne), and C. Wenske (Hannover) for their help with isotope analyses and Jürgen Koepke for help with microprobe analyses. The authors acknowledge the constructive comments by $\mathrm{E}$. Miller, J. Lee, and an anonymous reviewer that clarified and greatly improved the manuscript.

\section{References}

Adams, B. I., S. I. Wright, and K. Kunze (1993), Orientation imaging: The emergence of a new microscopy, Metall. Trans., 24A, 819-831.

Armstrong, R. L., and E. Hansen (1966), Cordilleran infrastructure in the eastern Great Basin, Am. J. Sci., 264, 112-127, doi:10.2475/ajs.264.2.112. 
Bartley, J. M., and B. P. Wernicke (1984), The Snake Range Décollement interpreted as a major extensional shear zone, Tectonics, 3, 647-657, doi:10.1029/TC003i006p00647.

Block, L., and L. Royden (1990), Core complex geometries and regional scale flow in the lower crust, Tectonics, 9, 557-567, doi:10.1029/ TC009i004p00557.

Bouchez, J.-L. (1977), Plastic deformation of quartzites at low temperature in an area of natural strain gradient, Tectonophysics, 39, 25-50, doi:10.1016/0040-1951(77)90086-5.

Chacko, T., H. Xiangsheng, K. M. Toshiko, R. N. Clayton, and J. R. Goldsmith (1996), Oxygen isotope fractionations in muscovite, phlogopite, and rutile, Geochim. Cosmochim. Acta, 60, 2595-2608, doi:10.1016/0016-7037(96)00112-3.

Cumbest, R. J., E. L. Johnson, and T. C. Onstott (1994), Argon composition of metamorphic fluids: Implications for ${ }^{40} \mathrm{Ar} /{ }^{39} \mathrm{Ar}$ geochronology, Geol. Soc. Am. Bull., 106, 942-951, doi:10.1130/0016-7606(1994) 106<0942:ACOMFI >2.3.CO;2.

Dingley, D. J., and D. P. Field (1997), Electron backscatter diffraction and orientation imaging microscopy, Mater. Sci. Technol., 13, 69-78.

Dunlap, W. J. (1992), Structure, kinematics, and cooling history of the Arltunga nappe complex, central Australia, Ph.D. thesis, Univ. of Minnesota, Minneapolis.

Famin, V., P. Philippot, L. Jolivet, and P. Agard (2004), Evolution of hydrothermal regime along a crustal shear zone, Tinos Island, Greece, Tectonics, 23, TC5004, doi:10.1029/2003TC001509.

Fricke, H. C., S. M. Wickham, and J. R. O'Neil (1992), Oxygen and hydrogen isotope evidence for meteoric water infiltration during mylonitization and uplift in the Ruby Mountains-East Humboldt Range core complex, Nevada, Contrib. Mineral. Petrol., 111, 203-221, doi:10.1007/ BF00348952.

Gans, P. B. (1987), An open system, two-layer crustal stretching model for the eastern Great Basin, Tectonics, 6, 1-12, doi:10.1029/ TC006i001p00001.

Gans, P. B., G. A. Mahood, and E. Schermer (1989), Synextensional Magmatism in the Basin and Range Province: A Case Study From the Eastern Great Basin, Geol. Soc. Am. Spec. Pap., 223, 53 pp.

Gans, P. B., and E. L. Miller (1983), Style of mid-Tertiary extension in east-central Nevada, Spec. Stud. Utah Geol. Miner. Surv., 59, 107-160.

Gans, P. B., E. L. Miller, J. McCarthy, and M. L. Oldcott (1985), Tertiary extensional faulting and evolving ductile-brittle transition zones in the northern Snake Range and vicinity: New insights from seismic data, Geology, 13, 189-193, doi:10.1130/0091-7613(1985)13<189: TEFAED $>2.0 . \mathrm{CO} ; 2$

Gans, P., E. L. Miller, and J. Lee (1999a), Preliminary Geology Map of the Spring Mountain 7.5' Quadrangle, Northern Snake Range, Nevada and Utah, Nev. Bur. Mines Geol. Field Stud. Map 18, Nev. Bur. Mines Geol., Reno.

Gans, P. B., E. L. Miller, C. C. Huggins, and J. Lee (1999b), Preliminary Geologic Map of the Little Horse Canyon 7.5' Quadrangle, Nevada and Utah, Nev. Bur. Mines Geol. Field Stud. Map 20, Nev. Bur. Mines Geol., Reno.

Gottardi, R., C. Teyssier, A. Mulch, T. W. Venneman, and M. L. Wells (2011), Preservation of an extreme transient geotherm in the Raft River detachment shear zone, Geology, 39, 759-762, doi:10.1130/G31834.1.

Heidelbach, F., K. Kunze, and H. R. Wenk (2000), Texture analysis of a recrystallised quartzite using electron diffraction in the scanning electron microscope, J. Struct. Geol., 22, 91-104, doi:10.1016/S0191-8141(99) 00125-X.

Hirth, G., and J. Tullis (1992), Dislocation creep regimes in quartz aggregates, J. Struct. Geol., 14, 145-159, doi:10.1016/0191-8141(92)90053-Y.

Holk, G. J., and H. P. Taylor Jr. (2007), ${ }^{18} \mathrm{O} /{ }^{16} \mathrm{O}$ Evidence for contrasting hydrothermal regimes involving magmatic and meteoric-hydrothermal waters at the Valhalla metamorphic core complex, British Columbia, Econ. Geol., 102, 1063-1078, doi:10.2113/gsecongeo.102.6.1063.

Jessell, M. W. (1987), Grain-boundary migration microstructures in a naturally deformed quartzite, J. Struct. Geol., 9, 1007-1014, doi:10.1016/ 0191-8141(87)90008-3.

Kirschner, D. L., M. A. Cosca, H. Masson, and J. C. Hunziker (1996), Staircase ${ }^{40} \mathrm{Ar} /{ }^{39} \mathrm{Ar}$ spectra of fine-grained white mica: Timing and duration of deformation and empirical constraints on argon diffusion, Geology, 24, 747-750, doi:10.1130/0091-7613(1996)024<0747:SAASOF>2.3.CO 2

Krieger Lassen, N. C. (1996), The relative precision of crystal orientations measured from electron backscattering patterns, J. Microsc., 181, 72-81, doi:10.1046/j.1365-2818.1996.95376.x.

Law, R. D. (1990), Crystallographic fabrics: A selective review of their applications to research in structural geology, in Deformation Mechanisms, Rheology and Tectonics, edited by R. J. Knipe and E. H. Rutter, Geol. Soc. Spec. Publ., 54, 335-352.
Lee, J. L. (1995), Rapid uplift and rotation of mylonitic rocks from beneath a detachment fault: Insights from potassium feldspar ${ }^{40} \mathrm{Ar} /{ }^{39} \mathrm{Ar}$ thermochronology, northern Snake Range, Nevada, Tectonics, 14, 54-77, doi:10.1029/94TC01508.

Lee, J., and J. F. Sutter (1991), Incremental ${ }^{40} \mathrm{Ar} /{ }^{39} \mathrm{Ar}$ thermochronology of mylonitic rocks from the Northern Snake Range, Nevada, Tectonics, 10 , 77-100, doi:10.1029/90TC01931.

Lee, D. E., R. F. Marvin, T. W. Stern, and Z. E. Peterman (1970), Modification of potassium-argon ages by Tertiary thrusting in the Snake Range, White Pine County, Nevada, U.S. Geol. Surv. Prof. Pap., $700-$ D, D92-D102.

Lee, D. E., R. F. Marvin, and H. H. Mehnert (1980), A radiometric age study of Mesozoic-Cenozoic metamorphism in eastern White Pine County, Nevada, and nearby Utah, U.S. Geol. Surv. Prof. Pap., 1158-C, 17-28.

Lee, J., E. L. Miller, and J. F. Sutter (1987), Ductile strain and metamorphism in an extensional tectonic setting: A case study from the northern Snake Range, Nevada, USA, in Continental Extensional Tectonics, edited by M. P. Coward, J. F. Dewey, and P. L. Hancock, Geol. Soc. Spec. Publ., 28, 267-298.

Lee, J., P. B. Gans, and E. L. Miller (1999a), Preliminary Geologic Map of the Mormon Jack Pass 7.5' Quadrangle, Northern Snake Range, White Pine County, Nev. Bur. Mines Geol. Field Stud. Map 17, Nev. Bur. Mines Geol., Reno.

Lee, J., P. B. Gans, and E. L. Miller (1999b), Preliminary Geologic Map of the Third Butte East 7.5' Quadrangle, Northern Snake Range, White Pine County, Nev. Bur. Mines Geol. Field Stud. Map 16, Nev. Bur. Mines Geol., Reno.

Lee, J., E. L. Miller, P. B. Gans, and C. C. Huggins (1999c), Preliminary Geologic Map of the Mount Moriah 7.5' Quadragle, Northern Snake Range, White Pine County, Nev. Bur. Mines Geol. Field Stud. Map 19, Nev. Bur. Mines Geol., Reno.

Lewis, C. J., B. P. Wernicke, J. Selverstone, and J. M. Bartley (1999), Deep burial of the footwall of the northern Snake Range decollement, Nevada, Geol. Soc. Am. Bull., 111, 39-51, doi:10.1130/0016-7606(1999) $111<0039:$ DBOTFO $>2.3 . \mathrm{CO} ; 2$

Lister, G. S., and A. W. Snoke (1984), S-C Mylonites, J. Struct. Geol., 6, 617-638, doi:10.1016/0191-8141(84)90001-4.

Lloyd, G. E., N. H. Schmidt, D. Mainprice, and D. J. Prior (1991), Crystallographic textures, Min. Mag., 55, 331-345, doi:10.1180/minmag. 1991.055.380.04

Losh, S. (1997), Stable isotope and modeling studies of fluid-rock interaction associated with the Snake Range and Mormon Peak detachment faults, Nevada, Geol. Soc. Am. Bull., 109, 300-323, doi:10.1130/00167606(1997) $109<0300:$ SIAMSO $>2.3$. CO;2

Mainprice, D. H., and M. S. Paterson (1984), Experimental studies of the role of water in the plasticity of quartzites, J. Geophys. Res., 89, 4257-4269, doi:10.1029/JB089iB06p04257.

Miller, E. L., and P. B. Gans (1989), Cretaceous crustal structure and metamorphism in the hinterland of the Sevier thrust belt, western U.S Cordillera, Geology, 17, 59-62, doi:10.1130/0091-7613(1989) 017<0059:CCSAMI>2.3.CO;2.

Miller, E. L., and P. B. Gans (1999), Preliminary Geologic Map of the Cove 7.5' Quadrangle, Northern Snake Range, White Pine County, Nev. Bur. Mines Geol. Field Stud. Map 22, Nev. Bur. Mines Geol., Reno.

Miller, E. L., P. B. Gans, and J. D. Garing (1983), The Snake Range decollement: An exhumed mid-Tertiary ductile-brittle transition, Tectonics, 2, 239-263, doi:10.1029/TC002i003p00239.

Miller, E. L., P. B. Gans, R. W. Brown, and T. A. Dumitru (1993), Apatite fission track evidence for Miocene extensional faulting, east-central Nevada, northern Basin and Range Province, Geol. Soc. Am. Abstr. Programs, 25, 121-122.

Miller, E. L., P. B. Gans, and S. P. Grier (1995), Geologic map of Windy Peak 7.5' quadrangle, White Pine County, Nevada, U.S. Geol. Surv. Open File Rep. OF94-687, scale 1:24,000.

Miller, E. L., T. A. Dumitru, R. W. Brown, and P. B. Gans (1999a), Rapid Miocene slip on the Snake Range-Deep Creek Range fault system, eastcentral Nevada, Geol. Soc. Am. Bull., 111, 886-905, doi:10.1130/00167606(1999) $111<0886:$ RMSOTS $>2.3$.CO;2.

Miller, E. L., P. B. Gans, S. P. Grier, C. C. Huggins, and J. Lee (1999b), Preliminary Geologic Map of the Old Mans Canyon 7.5' Quadrangle, Northern Snake Range, White Pine County, Nev. Bur. Mines Geol. Field Stud. Map 21, Nev. Bur. Mines Geol., Reno.

Mix, H., A. Mulch, and C. P. Chamberlain (2011), Cenozoic migration of topography in the North American Cordillera, Geology, 39, 87-90, doi:10.1130/G31450.1.

Morrison, J., and J. L. Anderson (1998), Footwall refrigeration along a detachment fault: Implications for the thermal evolution of core complexes, Science, 279, 63-66, doi:10.1126/science.279.5347.63. 
Mulch, A., and C. P. Chamberlain (2007), Stable isotope paleoaltimetry in orogenic belts-The silicate record in surface and crustal geological archives, Rev. Mineral. Geochem., 66, 89-118, doi:10.2138/rmg. 2007.66.4.

Mulch, A., and M. A. Cosca (2004), Recrystallization or cooling ages? In situ UV-laser 40Ar/39Ar geochronology of muscovite in mylonitic rocks, J. Geol. Soc., 161, 573-582, doi:10.1144/0016-764903-110.

Mulch, A., C. Teyssier, M. A. Cosca, O. Vanderhaeghe, and T. Vennemann (2004), Reconstructing paleoelevation in eroded orogens, Geology, 32, 525-528, doi:10.1130/G20394.1.

Mulch, A., M. A. Cosca, J. Fiebig, and A. Andresen (2005), Time scales of mylonitic deformation and meteoric fluid infiltration during extensional detachment faulting: An integrated in situ ${ }^{40} \mathrm{Ar} /{ }^{39} \mathrm{Ar}$ geochronology and stable isotope study of the Porsgrunn-Kristiansand Shear Zone (Southern Norway), Earth Planet. Sci. Lett., 233, 375-390, doi:10.1016/j.epsl. 2005.01.042.

Mulch, A., C. Teyssier, M. A. Cosca, and T. Vennemann (2006), Thermomechanical analysis of strain localization in a detachment zone, J. Geophys. Res., 111, B12405, doi:10.1029/2005JB004032.

Mulch, A., C. Teyssier, C. P. Chamberlain, and M. A. Cosca (2007), Stable isotope paleoaltimetry of Eocene Core Complexes in the North American Cordillera, Tectonics, 26, TC4001, doi:10.1029/2006TC001995.

Neumann, B. (2000), Texture development of recrystallised quartz polycrystals unravelled by orientation and misorientation characteristics, $J$. Struct. Geol., 22, 1695-1711, doi:10.1016/S0191-8141(00)00060-2.

Person, M., A. Mulch, C. Teyssier, and Y. Gao (2007), Isotope transport and exchange within metamorphic core complexes, Am. J. Sci., 307, 555-589, doi:10.2475/03.2007.01.

Poage, M. A., and C. P. Chamberlain (2001), Empirical relationships between elevation and the stable isotope composition of precipitation and surface waters: Considerations for studies of paleoelevation change, Am. J. Sci., 301, 1-15, doi:10.2475/ajs.301.1.1.

Rey, P., C. Teyssier, and D. L. Whitney (2009a), Extension rates, crustal melting, and core complex dynamics, Geology, 37, 391-394, doi:10.1130/G25460A.1.

Rey, P., C. Teyssier, and D. L. Whitney (2009b), The role of partial melting and extensional strain rates in the development of metamorphic core complexes, Tectonophysics, 477, 135-144, doi:10.1016/j.tecto.2009.03.010.

Rowley, D. B., and B. S. Currie (2006), Palaeo-altimetry of the late Eocene to Miocene Lunpola Basin, central Tibet, Nature, 439, 677-681, doi:10.1038/nature04506.

Rowley, D. B., and C. N. Garzione (2007), Stable isotope-based paleoaltimetry, Annu. Rev. Earth Planet. Sci., 35, 463-508, doi:10.1146/annurev. earth.35.031306.140155.

Schmidt, N. H., and N. O. Olesen (1989), Computer-aided determination of crystal-lattice orientation from electron-channeling patterns in the SEM, Can. Mineral., 27, 15-22.
Sheppard, S. M. F. (1986), Characterization and isotopic variations in natural waters, Rev. Mineral., 16, 165-184.

Suzuoki, T., and S. Epstein (1976), Hydrogen isotope fractionation between $\mathrm{OH}$-bearing minerals and water, Geochim. Cosmochim. Acta, 40, 1229-1240, doi:10.1016/0016-7037(76)90158-7.

ten Grotenhuis, S. M., R. A. J. Trouw, and C. W. Passchier (2003), Evolution of mica fish in mylonitic rocks, Tectonophysics, 372, 1-21, doi:10.1016/S0040-1951(03)00231-2.

Teyssier, C., E. C. Ferré, D. L. Whitney, B. Norlander, O. Vanderhaeghe, and D. Parkinson (2005), Flow of partially molten crust and origin of detachments during collapse of the Cordilleran Orogen, in High Strain Zones: Structure and Physical Properties, edited by D. Bruhn and L. Burlini, Geol. Soc. Spec. Publ., 245, 39-64, doi:10.1144/GSL. SP.2005.245.01.03.

Tirel, C., J. P. Brun, and E. Burov (2008), Dynamics and structural development of metamorphic core complexes, J. Geophys. Res., 113, B04403, doi:10.1029/2005JB003694.

Tullis, J., J. M. Christie, and D. T. Griggs (1973), Microstructures and preferred orientations in experimentally deformed quartzites, Geol. Soc. Am. Bull., 84, 297-314, doi:10.1130/0016-7606(1973)84<297: MAPOOE $>2.0 . \mathrm{CO} ; 2$

Wernicke, B. (1981), Low-angle normal faults in the Basin and Range Province: Nappe tectonics in an extending orogen, Nature, 291, 645-648, doi:10.1038/291645a0.

Wickham, S. M., and H. P. Taylor (1987), Stable isotope constraints on the origin and depth of penetration of hydrothermal fluids associated with Hercynian regional metamorphism and crustal anatexis in the Pyrenees, Contrib. Mineral. Petrol., 95, 255-268, doi:10.1007/BF00371841.

Wijbrans, J. R., and I. Mc Dougall (1986), 40Ar/39Ar dating of white micas from an Alpine high-pressure metamorphic belt on Naxos (Greece): The resetting of the argon isotopic system, Contrib. Mineral. Petrol., 93, 187-194, doi:10.1007/BF00371320.

Wilson, C. J. L., and I. A. Bell (1979), Deformation of biotite and muscovite, Tectonophysics, 58, 179-200, doi:10.1016/0040-1951(79)90328-7.

A. Gébelin and A. Mulch, Biodiversity and Climate Research Centre (BiK-F), Senckenberganlage 25, D-60325 Frankfurt am Main, Germany. (aude.gebelin@senckenberg.de)

M. Heizler, New Mexico Bureau of Geology and Mineral Resources, New Mexico Institute of Mining and Technology, 801 Leroy P1., Socorro, NM 87801, USA.

N. C. A. Seaton and C. Teyssier, Department of Geology and Geophysics, University of Minnesota, 310 Pillsbury Dr., Minneapolis, MN 55455, USA.

T. Vennemann, Institute of Mineralogy and Geochemistry, University of Lausanne, BFSH-2, Lausanne CH-1015, Switzerland. 